\title{
A Review on the Modified Finite Point Method
}

\author{
Nan-Jing Wu, ${ }^{1}$ Boe Shiun Chen, ${ }^{2}$ and Ting-Kuei Tsay ${ }^{2}$ \\ ${ }^{1}$ Tainan Hydraulics Laboratory, National Cheng Kung University, Tainan City 70955, Taiwan \\ ${ }^{2}$ Department of Civil Engineering, National Taiwan University, Taipei City 10617, Taiwan \\ Correspondence should be addressed to Ting-Kuei Tsay; tktsay@ntu.edu.tw
}

Received 29 December 2013; Accepted 30 May 2014; Published 24 July 2014

Academic Editor: Keith Ng

Copyright (C) 2014 Nan-Jing Wu et al. This is an open access article distributed under the Creative Commons Attribution License, which permits unrestricted use, distribution, and reproduction in any medium, provided the original work is properly cited.

The objective of this paper is to make a review on recent advancements of the modified finite point method, named MFPM hereafter. This MFPM method is developed for solving general partial differential equations. Benchmark examples of employing this method to solve Laplace, Poisson, convection-diffusion, Helmholtz, mild-slope, and extended mild-slope equations are verified and then illustrated in fluid flow problems. Application of MFPM to numerical generation of orthogonal grids, which is governed by Laplace equation, is also demonstrated.

\section{Introduction}

In recent decades, a new type of numerical methods for solving partial differential equations (PDEs) without meshes has been developed. These methods are called meshless or meshfree methods, which refer to the idea that information of connectivity of each mesh or element is no longer required as the input data in this new development. It saves a lot of manpower in the tedious preprocessing stage in preparing numerical computations. However, the information of the discretized nodes, such as the position vectors, is still a necessary requirement.

The idea of meshless methods can be traced back to the smoothed particle hydrodynamics (SPH) for modeling the astrophysical phenomenon [1]. The basic concept of meshless methods is to approximate the solution of a PDE as a linear combination of basis functions or shape functions. The process for solving the PDE is to seek the undetermined factors which are mostly just coefficients of the basis functions and in some other cases could be the values of the solution itself at discretized nodes.

Meshless methods are categorized mainly into two parts [2], namely, methods based on weak-form formulations and methods based on collocation techniques, also known as the strong-form methods. Though strong-form methods are much simpler and more straightforward, it was reported in [2] that there has been less research devoted to strong-form methods. This is partly because they are less robust and partly because researches were concentrated on the finite element method (FEM) which used weak-forms. It was then a natural step to favor the use of weak-form methods.

In the 1990s, a general collocation method to solve linear partial differential equations was proposed by adopting the radial basis functions (RBFs) [3]. There are two kinds of RBF collocation methods, domain-type and boundary-type. The application of domain-type RBF collocation methods is broader, including tissue engineering $[4,5]$, heat transfer [6], nonlinear Burgers' equations [7], shallow water equations [8-10], Stokes flows [11], electromagnetic problems [12, 13], and finance $[14,15]$. However, it was reported that domaintype RBF collocation methods usually malfunction in the seeking of partial derivatives around the boundaries $[16,17]$. On the contrary, boundary-type RBF collocation methods, in which governing equations are satisfied automatically in the entire domain, are more applicable to problems in which accurate partial derivatives of the solutions around the boundaries are desirable. Typical examples are the application of MFS (method of fundamental solutions) to water wave problems [18-20]. Nevertheless, boundary-type RBF collocation methods are limited to problems governed by some specific equations, such as Laplace or Helmholtz equations. In [21], a modified domain-type RBF collocation method was proposed. With additional satisfaction to the governing equation on boundary collocation points, the gradient of 
the velocity potential at any free surface node, which represents the velocity vector at that specific node, was accurately estimated. Thus this method was successfully applied to the simulation of water waves in a swaying tank.

Apart from the combination of RBFs, one could use a polynomial to approximate the solution of a PDE and seek the coefficients of all the monomials by applying the collocation technique. However, polynomials could not be used in a global solution form such as what is usually did in RBF collocation methods. This is because the values of high degree terms could become extremely large at points far from the origin. In most polynomial collocation methods, polynomials are just applied to approximate the solution piece-wisely around discretized nodes. The moving least square (MLS) or weighted least square (WLS) approaches are always accompanied by the polynomial approximation to transform and combine the locally approximated solutions into a global solution form. In each local solution, basis functions are the monomials and their factors are just the coefficients. The least square (LS) approach with Taylor series expansion was developed for the finite difference with arbitrary meshes [22] while the WLS and MLS are introduced to the polynomial collocation methods in $[23,24]$ which stated that in most cases local approximation with just LS is not stable. The WLS and MLS are identical to each other when they are used for the local approximation at discretized nodes. The difference is on how to calculate the partial derivatives of the approximation. In [23] one could find that both the performances of MLS and WLS are acceptable.

In the global solution form, the basis functions are called the shape functions and their factors are the values of the solution itself. This localizing approach is somewhat like localized RBF collocation methods [25]. Representatives of this family are the $h p$-meshless cloud method [26] and the finite point method (FPM) [23, 24].

Like most strong-form methods, collocation methods with local polynomial approximation are reported to be unstable [27] and usually can work only for problems with simple and regular geometry and boundary conditions. This coincides with what was reported in [2]. Contrarily, weak-form methods with MLS or WLS approaches [2835], which need background cells for local integration, yield much higher stability and are applied more widely in many numerical implementations, such as viscous flow problems [31], nonlinear water wave problems [32, 33], and wave breaking problems $[34,35]$. In some studies efforts are made to generalize the FPM to weak-form formulation by applying alternative weights [36] or alternative derivative approximation $[37,38]$ in the FPM. A new meshless method, based on subdomain collocation approach, is also present and named "the generalized finite point method" (GFPM) [38].

Though sometimes unstable, the simplicity renders the strong-form methods worthy of further investigations. Studies on improving the collocation techniques and the construction of local clouds, which means the selection of neighboring nodes involved in the local approximation, are important issues for increasing the numerical stability [39-41].

Besides methods mentioned above, one could also identify a few literatures about meshless methods employing the reproducing kernel approximation [42-46]. With complicated formulation, these methods accurately approximate PDE's solutions as linear combinations of shape functions in a global way. In [44] it was reported that "satisfying the boundary conditions and the governing equations simultaneously at the boundary can provide a more accurate solution to a given problem." This coincides with what was reported in [21]. Improvements of the approximation for making the governing equation satisfied at the boundaries were also proposed in [44]. Though in polynomial collocation methods with MLS approach the global solution forms are also the linear combinations of shape functions, the basic concept differs from the reproducing kernel approximation so that the way for collocation is not the same. In methods with reproducing kernel approximation, such as finite cloud methods (FCM), the partial derivatives of the solution are obtained from the differentiation of the global shape functions while in collocation methods with local polynomial approximation they are acquired by differentiating the local basis functions, which could turn out to be just the coefficients of higher monomials as part of the numerical solutions when the WLS approach is employed.

Adopting the advantage of localization, a robust local polynomial collocation method, which is similar to some finite point methods in which polynomials are localized by placing their origins at collocation points [39-41], was proposed in [47]. Inspired by [21, 44], the satisfaction of the governing equation is additionally required on boundary collocation points. This makes the method proposed in [47] not just as simple and straightforward as other similar methods, but also more robust and stable. Because this method mainly originates from the finite point method but improved, we hereby nominate it as the modified finite point method (MFPM). This method has been employed to several engineering and science applications [21, 48-53]. This paper is to review the modified finite point method, particularly emphasizing on its improvements and recent findings. Verifications of MFPM are illustrated in benchmark problems, and its recent applications to engineering and science problems are also demonstrated.

\section{Formulations of Modified Finite Point Method}

The local polynomial collocation method proposed in [47], which originates from the finite point method, is a meshfree method for solving general partial differential equations (PDEs). A mesh-free method does not require the connectivity of nodal points of a mesh or element. Instead, the position vectors of computational nodes are the only information required to be provided as the input in the preprocessing stage of computations. This advantage of mesh-free methods renders them increasingly popularity in selection of numerical methods.

Taking a general 2-D linear second order PDE as an example, the descriptions of this method and the conventional method are given as follows. 
2.1. The Local Approximation. When solving a general 2-D linear, second order PDE is

$$
\begin{gathered}
\mathscr{L}\{\phi\}=c_{1} \phi+c_{2} \frac{\partial \phi}{\partial x}+c_{3} \frac{\partial \phi}{\partial y}+c_{4} \frac{\partial^{2} \phi}{\partial x^{2}} \\
+c_{5} \frac{\partial^{2} \phi}{\partial y^{2}}+c_{6} \frac{\partial^{2} \phi}{\partial x \partial y}=s
\end{gathered}
$$

subjected to specified boundary conditions

$$
\begin{gathered}
\mathscr{B}\{\phi\}=q_{1} \phi+q_{2} \frac{\partial \phi}{\partial x}+q_{3} \frac{\partial \phi}{\partial y}=f \\
\vec{x} \in \Gamma_{1}, \\
\phi=\phi_{b}, \quad \vec{x} \in \Gamma_{2},
\end{gathered}
$$

where $c_{1}, c_{2}, \ldots, c_{6}, q_{1}, q_{2}, q_{3}, f$, and $s$ are all functions of $x, y$ and may change in time in transient problems. The boundary $\Gamma_{1}$ could be nonsmooth and then at a corner there could be more than one condition like (2). Therefore, $q_{1}, q_{2}, q_{3}$, and $f$ could be multivalued in this case. Boundary condition expressed as (2) is just for conciseness. It will be explained later on how boundary conditions will be treated at a point where more than one boundary condition exists. In seeking the numerical solutions, the entire domain is distributed with $N$ nodes as needed. At each node, $\vec{x}=\vec{x}_{j}$, $\phi$ is approximated as

$$
\left.\phi(\vec{x})\right|_{\vec{x} \approx \vec{x}_{j}} \approx \widehat{\phi}_{j}(\vec{x})=\sum_{i=1}^{m} \alpha_{j i} p_{i}(\vec{X})
$$

in which $\vec{X}=\vec{x}-\vec{x}_{j}$ is the relative position vector, $p_{i}(\vec{X})$ is the $i$ th monomial of the polynomial, and $\alpha_{j i}$ are coefficients to be determined. The subscript $j$ indicates that this approximation is valid only in the vicinity of $\vec{x}_{j}$. Once a new $\vec{x}_{j}$ is chosen, there will be a new set of $\alpha_{j i}$. For a 2$\mathrm{D}$ problem governed by a second order partial differential equation, the monomials are

$$
\begin{aligned}
& \left\{p_{i}(\vec{X}), i=1 \sim m\right\} \\
& \quad=\left\{\begin{array}{llllllllllll}
1 & X & Y & X^{2} & Y^{2} & X Y & X^{3} & X^{2} Y & X Y^{2} & Y^{3} & \cdots
\end{array}\right\}
\end{aligned}
$$

in which $\vec{X}=X \vec{i}+Y \vec{j}$. The value of $m$ which represents that the chosen degree of the polynomial should be at least the same as the degree of the polynomial. It has been suggested [47] that the degree of the polynomial higher than the order of the differential equation by one might be better. The approximations of the solution and its partial derivatives are just formulated simply by the local approximation. In 2-D problems for example, they are

$$
\left.\left.\phi\right|_{\vec{x}=\vec{x}_{j}} \approx \hat{\phi}\right|_{\vec{x}_{\bar{x}} \vec{x}_{j}}=\alpha_{j 1},\left.\left.\quad \frac{\partial \phi}{\partial x}\right|_{\vec{x}_{\vec{x}} \vec{x}_{j}} \approx \frac{\partial \widehat{\phi}}{\partial x}\right|_{\vec{x}=\vec{x}_{j}}=\alpha_{j 2}
$$

$$
\begin{gathered}
\left.\left.\frac{\partial \phi}{\partial y}\right|_{\vec{x}=\vec{x}_{j}} \approx \frac{\partial \hat{\phi}}{\partial y}\right|_{\vec{x}=\vec{x}_{j}}=\alpha_{j 3}, \\
\left.\left.\frac{\partial^{2} \phi}{\partial x^{2}}\right|_{\vec{x}_{=\vec{x}_{j}}} \approx \frac{\partial^{2} \hat{\phi}}{\partial x^{2}}\right|_{\vec{x}=\vec{x}_{j}}=2 \alpha_{j 4}, \\
\left.\left.\frac{\partial^{2} \phi}{\partial y^{2}}\right|_{\vec{x}_{=\vec{x}_{j}}} \approx \frac{\partial^{2} \hat{\phi}}{\partial y^{2}}\right|_{\vec{x}_{x}=\vec{x}_{j}}=2 \alpha_{j 5},
\end{gathered}
$$$$
\left.\left.\frac{\partial^{2} \phi}{\partial x \partial y}\right|_{\vec{x}_{=\vec{x}_{j}}} \approx \frac{\partial^{2} \hat{\phi}}{\partial x \partial y}\right|_{\vec{x}=\vec{x}_{j}}=\alpha_{j 6}, \ldots
$$

Here the error residual of the local approximation around $\vec{x}=\vec{x}_{j}$ is defined as

$$
E_{j}=\sum_{l=1}^{N}\left(W_{j l}\left(\phi\left(\vec{x}_{l}\right)-\widehat{\phi}_{j}\left(\vec{x}_{l}\right)\right)^{2}\right)
$$

where $W_{j l}$ is a weighting factor determined by the distance between $\vec{x}_{j}$ and $\vec{x}_{l}$. Usually, a normalized Gaussian function is selected for determining the weighting factor

$$
W_{j l}=\left\{\begin{array}{lc}
\frac{\exp \left(-\varepsilon\left(r_{j l} / \rho_{j}\right)^{2}\right)-\exp (-\varepsilon)}{1-\exp (-\varepsilon)}, & r_{j l}<\rho_{j} \\
0, & r_{j l} \geq \rho_{j},
\end{array}\right.
$$

where $r_{j l}$ is the distance between $\vec{x}_{j}$ and $\vec{x}_{l}$ (i.e., $r_{j l}=\vec{x}_{l}-$ $\left.\vec{x}_{j} \mid\right), \varepsilon$ is the shape parameter, and $\rho_{j}$ is the supporting range measured from the point of $\vec{x}_{j}$. The weight, $W_{j l}$, is intended to keep this parameter roughly within a range of optimal choice, regardless of the physical size of computational domain. Considering only the nonzero terms, (7) can be rewritten as

$$
E_{j}=\sum_{k=1}^{n}\left(W_{j k}\left(\phi\left(\vec{x}_{k}\right)-\widehat{\phi}_{j}\left(\vec{x}_{k}\right)\right)^{2}\right),
$$

where $k$ is the local index number of $\vec{x}_{l}$ in the $j$ th subdomain and $n$ is number of nodes inside the subdomain for local collocation. The nodal index relationship between local and global domains is illustrated in Figure 1. Other alternative functions for considering the value of the weight can be found in references of the meshless methods using the MLS or WLS approach, both in weak-form and strong-form formulations [22-24, 26-41]. The weights are treated as functions in the MLS approach while they are just factors in seeking the approximations of the derivatives when WLS approach is employed.

2.2. The Conventional Collocation Method. Since the modified finite point method is originated from the conventional finite point method, the collocation method in the conventional finite point method will be presented briefly for completeness in this section. 


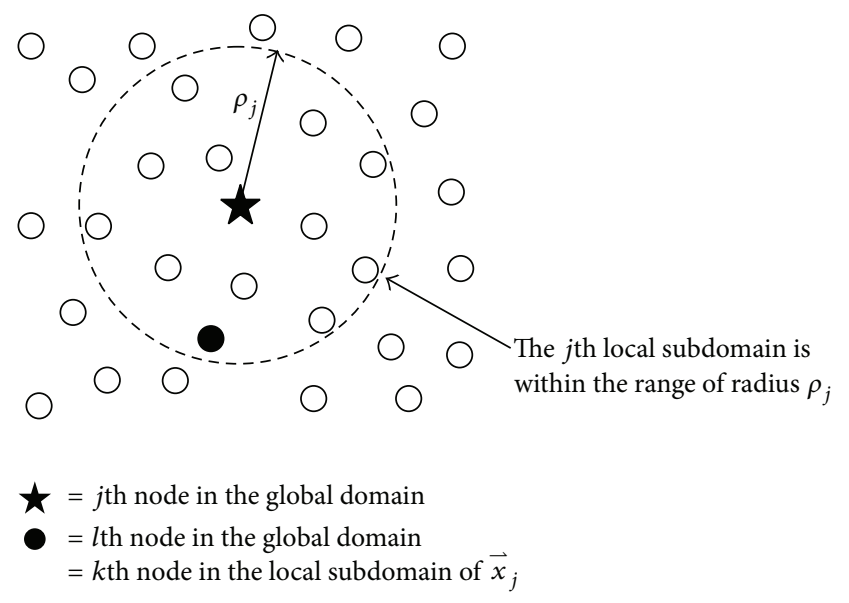

FIGURE 1: Illustration of the nodal index relationship between local and global domains.

The coefficients $\alpha_{j i}$ corresponding to the minimized $E_{j}$ in (9) can be expressed explicitly by the least square approach as

$$
\left[\alpha_{j}\right]=[\Lambda][\beta]
$$

in which

$$
\begin{gathered}
{\left[\alpha_{j}\right]_{m \times 1}=\left[\begin{array}{lllll}
\alpha_{j 1} & \cdots & \alpha_{j i} & \cdots & \alpha_{j m}
\end{array}\right]^{T}} \\
{[\Lambda]_{m \times n}=\left([A]^{T}[A]\right)^{-1}[A]^{T}} \\
{[A]_{n \times m}=\left[\begin{array}{ccccc}
a_{11} & a_{12} & \cdots & \cdots & a_{1 m} \\
a_{21} & \ddots & & & a_{2 m} \\
\vdots & & a_{k i} & & \vdots \\
\vdots & & & \ddots & \vdots \\
a_{n 1} & a_{n 2} & \cdots & \cdots & a_{n m}
\end{array}\right]} \\
{[\beta]_{n \times 1}=\left[\begin{array}{llllll}
\beta_{1} & \cdots & \beta_{k} & \cdots & \beta_{n}
\end{array}\right]^{T}} \\
=\left[\begin{array}{lllll}
w_{1} \phi_{1} & \cdots & w_{k} \phi_{k} & \cdots & w_{n} \phi_{n}
\end{array}\right]^{T},
\end{gathered}
$$

where $w_{k}=\sqrt{W_{j k}}, \phi_{k}=\phi\left(\vec{x}_{k}\right)$, and $a_{k i}=w_{k} p_{i}\left(\vec{x}_{k}-\vec{x}_{j}\right)$.

It should be noted that this approximation is only valid in the vicinity of $\vec{x}_{j}$. Once a new $\vec{x}_{j}$ is chosen, the entries of matrix $[\Lambda]$ and components of vector $[\beta]$ should all be renewed.

When employing a conventional collocation method to solve a PDE, what one needs to do is just use the approximations of the solution and its partial derivatives to formulate a global matrix system and solve it. In the process of collocation, because it is anticipated to find local approximations of the solution and its partial derivatives very close to the relevant exact values which are not known a priori, the approximations listed in (6) are deemed as the exact values. This is similar to what is done in finite difference methods (FDM). Therefore, one gets a global matrix equation when assembling the local approximations into the global system

$$
[K]_{N \times N}[\phi]_{N \times 1}=[b]_{N \times 1} \text {. }
$$

In the case that $\vec{x}_{j}$ is on the boundary and the value of $\phi$ is known, the entities in (12) are

$$
\begin{gathered}
\kappa_{j l}= \begin{cases}1, & \text { if } j=l \\
0, & \text { otherwise }\end{cases} \\
b_{j}=\phi_{j} .
\end{gathered}
$$

When the collocation point is inside the domain, the entities in (12) are

$$
\kappa_{j l}=\left\{\begin{array}{cl}
w_{k}\left(c_{1} \lambda_{1 k}+c_{2} \lambda_{2 k}+c_{3} \lambda_{3 k}+2 c_{4} \lambda_{4 k}\right. & \\
\left.+2 c_{5} \lambda_{5 k}+c_{6} \lambda_{6 k}\right)_{\vec{x}=\vec{x}_{j}}, & \text { if }\left|\vec{x}_{j}-\vec{x}_{l}\right|<\rho_{j} \\
0, & \text { otherwise } \\
b_{j}=\left.s\right|_{\vec{x}=\vec{x}_{j}}, &
\end{array}\right.
$$

where $\lambda_{i k}$ indicates the $i$ th entry in $k$ th column in matrix $[\Lambda]$. When the collocation point is on the boundary where a Neumann condition or a Robin condition is given, the entities in (12) are

$$
\begin{gathered}
\kappa_{j l}= \begin{cases}w_{k}\left(q_{1} \lambda_{1 k}+q_{2} \lambda_{2 k}+q_{3} \lambda_{3 k}\right)_{\vec{x}=\vec{x}_{j}}, & \text { if }\left|\vec{x}_{j}-\vec{x}_{l}\right|<\rho_{j} \\
0, & \text { otherwise }\end{cases} \\
b_{j}=\left.f\right|_{\vec{x}=\vec{x}_{j}} .
\end{gathered}
$$

2.3. The Modified Collocation Method. In the conventional method, when the collocation point is on the boundary, the satisfactory of the governing equation is not used. When the collocation point is on the boundary where two or more conditions are given, only one of them is chosen for the conventional collocation. The partial derivatives are thus inaccurately estimated as a result of lacking the complete satisfaction of all equations. Inspired by [21, 44], an alternative local approximation for direct collocation was proposed in [47]. The coefficients of the local polynomial corresponding to the minimal error residual at the node $\vec{x}_{j}$ (9) under the condition that

$$
\begin{aligned}
(\mathscr{L}\{\phi\}-s)^{2}+\left(\mathscr{B}_{1}\{\phi\}-f_{1}\right)^{2} & \\
& +\cdots+\left(\mathscr{B}_{n_{\text {nd }}}\{\phi\}-f_{n_{\text {nd }}}\right)^{2} \longrightarrow 0
\end{aligned}
$$

where $n_{\text {nd }}$ is the number of non-Dirichlet boundary conditions at the node $\vec{x}=\vec{x}_{j}$, can be expressed as

$$
\left[\alpha_{j}\right]=\left[\Lambda^{\prime}\right]\left[\begin{array}{l}
\beta \\
\beta^{\prime}
\end{array}\right]
$$


in which

$$
\begin{gathered}
{\left[\Lambda^{\prime}\right]_{m \times\left(n+n_{\mathrm{nd}}+1\right)}=\left(\left[\begin{array}{c}
A \\
A^{\prime}
\end{array}\right]^{T}\left[\begin{array}{c}
A \\
A^{\prime}
\end{array}\right]\right)^{-1}\left[\begin{array}{c}
A \\
A^{\prime}
\end{array}\right]^{T}} \\
{\left[A^{\prime}\right]_{\left(n_{\mathrm{nd}}+1\right) \times m}} \\
=\left[\begin{array}{cccccccc}
w^{\prime} c_{1} & \cdots & \cdots & \cdots & w^{\prime} c_{p} & 0 & \cdots & 0 \\
w^{\prime} q_{1,1} & \cdots & w^{\prime} q_{1,3} & 0 & \cdots & \cdots & \cdots & 0 \\
\vdots & & \vdots & \vdots & & & & \vdots \\
w^{\prime} q_{n_{\mathrm{nd}}, 1} & \cdots & w^{\prime} q_{n_{\mathrm{nd}}, 3} & 0 & \cdots & \cdots & \cdots & 0
\end{array}\right] \\
{\left[\beta^{\prime}\right]_{\left(n_{\mathrm{nd}}+1\right) \times 1}=\left[\begin{array}{lllllll}
w^{\prime} s & w^{\prime} f_{1} & \cdots & w^{\prime} f_{n_{\mathrm{nd}}}
\end{array}\right]^{T},}
\end{gathered}
$$

where the definitions of $w_{k}, \phi_{k}, a_{k i}$ are all the same as in the conventional collocation method, while $w^{\prime}=\sqrt{W^{\prime}}$ in which $W^{\prime}$ represents a penalty weighting factor whose value is much greater than 1 . In the case of $n_{\text {nd }}$ in (17) is greater than 1 , it obviously indicates that the collocation point rests on an edge or at a corner. At an interior node, there is only one term in (13) (i.e., $n_{\text {nd }}=0$ ).

Assembling the local approximations into a global matrix system, one also gets a global matrix equation as (12), but the entries in this matrix equation are different from those described in (13)-(16), except those specified by the Dirichlet boundary condition. At all other points no matter inside the domain or on the boundaries, where non-Dirichlet conditions are given, the entries in (12) are

$$
\begin{gathered}
\kappa_{j l}= \begin{cases}w_{k} \lambda_{1 k}^{\prime}-\delta_{j}(l), & \text { if }\left|\vec{x}_{j}-\vec{x}_{l}\right|<\rho_{j} \\
0, & \text { otherwise }\end{cases} \\
\delta_{j}(l)= \begin{cases}1, & \text { if } j=l \\
0, & \text { otherwise }\end{cases} \\
b_{j}=-\sum_{k=1}^{n_{\text {nd }}+1} \lambda_{1,(n+k)}^{\prime} \beta_{k}^{\prime},
\end{gathered}
$$

where $\lambda_{1 k}^{\prime}$ indicates the first entry in kth column in matrix $\left[\Lambda^{\prime}\right]$.

2.4. The Parameters. Both in the conventional finite point method and in the modified one, there are several parameters to be chosen. They are the shape parameter $\varepsilon$ in the normalized Gaussian function as the weight, the subdomain size, $\rho_{j}$, and the degree of polynomial for the local approximation. The penalty weighting factor $W^{\prime}$ needs to be additionally given in MFPM. In [47] the shape parameter $\varepsilon$ in the normalized Gaussian function for the modified method has been shown to be much more insensitive than the one needed in the conventional method. It was suggested that $\varepsilon=22, \rho_{j}$ as 1.05 times of the distance from $\vec{x}_{j}$ to its 25th nearest neighboring node, and $W^{\prime}=10^{4}$ be chosen for numerical computations in different problems in this paper. The third degree polynomial gives better performance in both the conventional and the modified method for second order partial differential equations.

\section{Verifications of Benchmark Problems}

In this section, the following problems of different form of partial differential equations are chosen as benchmark problems to verify the accuracy of MFPM.

3.1. Laplace Equation. A potential uniform flow passing around a single cylinder in an infinite 2-D domain is chosen as the benchmark. The radius of the cylinder is $a$. Viscous effects which could be significant in real fluids are not considered here. The velocity at $x= \pm \infty$ or $y= \pm \infty$ is $\vec{v}=U \vec{i}+0 \vec{j}$. The velocity potential $\phi$ is governed by the Laplace equation:

$$
\frac{\partial^{2} \phi}{\partial x^{2}}+\frac{\partial^{2} \phi}{\partial y^{2}}=0
$$

The velocity field is determined as $\vec{v}=u \vec{i}+v \vec{j}$ :

$$
\begin{aligned}
& u=\frac{\partial \phi}{\partial x} \\
& v=\frac{\partial \phi}{\partial y} .
\end{aligned}
$$

There is an exact solution expressed as

$$
\phi_{e}=U x\left(1+\frac{a^{2}}{x^{2}+y^{2}}\right) .
$$

The subscript " $e$ " denotes the "exact solution."

Due to symmetry, only the upper left quadrant is modeled. The values of $U$ and $a$ are chosen as 1.0 and 0.5 , respectively. These quantities are dimensionless. The computational domain is within the range of $-2 \leq x \leq 0$ and $0 \leq$ $y \leq 2$. The specification of the boundary conditions and the arrangement of the collocation points are shown in Figure 2. Two nodal arrangements shown in Figure 3 are chosen for the verifications. The velocity potential at the upper boundary in Figure 2 is specified by the analytical solutions along this boundary. No-flux boundary condition is applied to the lower boundary, due to symmetry of the problem on the straight line and physical solid boundary on the quad-circle curve, respectively.

The profile of computed flow speed $\left(V=\sqrt{u^{2}+v^{2}}\right)$ along the cylinder surface is shown in Figure 4 . It is obvious that the modified method performs much better than the conventional one. In the comparison between these two panels in Figure 4, one could find that nodal distribution is much less sensitive in the modified method than in the conventional one. In the right panel of Figure 4, the result by using the conventional collocation method is actually acceptable. This indicates that the conventional collocation method functions properly when the nodes are carefully arranged. This observation coincides with what was reported in [29]: polynomial collocation methods with MLS approach usually can work only for problems with simple and regular geometry and boundary conditions. It should also be noted that, at $\theta=\pi$, which is a stagnation point, the result 


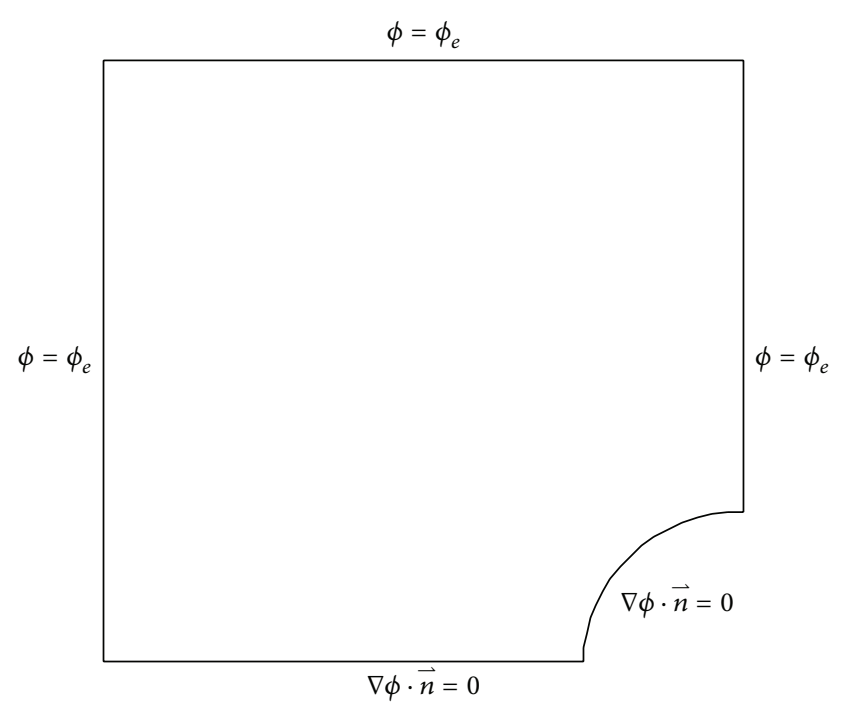

FIGURE 2: Boundary conditions for a problem of uniform flow passing a circular cylinder.

of the MFPM solution is exact while the result of the conventional collocation method is incorrect. This is because in the conventional collocation method only one Neumann boundary condition is employed in the collocation but in the MFPM the Neumann boundary conditions of both directions and the satisfactory of the governing equation are all used. This comparison has been done in [47]. Here we replot the figures and put them in the corresponding positions for the convenience of comparison.

3.2. Poisson Equation. Consider the following 2-D Poison equation:

$$
\frac{\partial^{2} \phi}{\partial x^{2}}+\frac{\partial^{2} \phi}{\partial y^{2}}=\sin (\pi x) \sin (\pi y)
$$

within the domain of $0 \leq x \leq 1,0 \leq y \leq 1$. The boundary conditions are

$$
\begin{gathered}
\frac{\partial \phi}{\partial y}=\frac{-1}{2 \pi} \sin (\pi x) \cos (\pi y), \quad \text { along } y=0, y=1 \\
\phi=0, \quad \text { along } x=0, x=1 .
\end{gathered}
$$

The exact solution of this Poisson equation can be found as $\phi_{e}=-\sin (\pi x) \sin (\pi y) /\left(2 \pi^{2}\right)$.

The verification of the MFPM to this problem was demonstrated in [47]. Here we redo this problem by increasing the nodal density and plot the contours of the numerical result together with the exact solution for the convenience of comparison. A uniform nodal arrangement is chosen for numerical computations of this problem. The nodal spacing is 0.05 . Therefore, totally 441 nodes are used in this test. The comparison of the numerical results with the analytical solutions is shown in Figure 5.
3.3. Convection-Diffusion Equation. Consider the following steady-state convection-diffusion equation [54]:

$$
\frac{\partial^{2} \phi}{\partial x^{2}}+\frac{\partial^{2} \phi}{\partial y^{2}}-U \frac{\partial \phi}{\partial x}=0
$$

in the region of $0 \leq x \leq 1,0 \leq y \leq 0.6$,

with boundary conditions:

$$
\begin{array}{cc}
\left.\phi\right|_{x=0}=1, & \left.\phi\right|_{x=1}=2, \\
\left.\frac{\partial \phi}{\partial y}\right|_{y=0}=0, & \left.\frac{\partial \phi}{\partial y}\right|_{y=0.6}=0 .
\end{array}
$$

This problem has an exact solution as

$$
\phi_{e}=2-\frac{1-\exp [U(x-1)]}{1-\exp (-U)} .
$$

A strong convective case of $U=100$ is chosen. The nodal arrangement for this problem is shown in Figure 6. The nodal spacing is mainly $\Delta x=\Delta y=0.02$ while near the right boundary the nodal density is increased to $\Delta x=\Delta y=0.01$, in order to catch the behavior of exponential growth near the boundary of $x=1$. Totally 1,825 nodes are used in this test. Only $1.48 \%$ of the entries in the global matrix $[K]$ are nonzero. Profiles of $\phi$ and $\partial \phi / \partial x$ along $y=0$ and $y=0.3$ are shown in Figure 7. Very good agreement between present numerical results and the exact solutions of function values and its partial derivatives is obtained. The verification of the MFPM to this problem was demonstrated in [47]. Here we replot the figures of this problem for the convenience of comparison.

3.4. Helmholtz Equation. In previous three benchmark problems, unknowns of real-valued governing equations are dealt with. There are physical problems governed by equation with complex-valued unknowns. It is intended to choose freesurface water waves problems to demonstrate applicability of MFPM to such problems.

In the constant water depth, the motion of progressive, monochromatic free-surface waves can be reduced to a 2-D problem governed by Helmholtz equation

$$
\frac{\partial^{2} \phi}{\partial x^{2}}+\frac{\partial^{2} \phi}{\partial y^{2}}+k^{2} \phi=0
$$

where $\phi$, a complex valued variable, is the pseudo velocity potential of the fluid on the free surface. The symbols used in physical problems hereafter should be differentiated from those used in the formulation of the MFPM. Wave number $k$ is defined as $2 \pi / L$, in which $L$ is the wavelength of the monochromatic waves. The real free-surface velocity potential of monochromatic waves is defined as

$$
\Phi=\operatorname{Re}\left\{\phi e^{-i \omega t}\right\}
$$

in which $i=\sqrt{-1}$ is the pure imaginary number. The symbol $\omega$ indicating the angular frequency is defined as $\omega=2 \pi / T$ and $T$ is the period of the monochromatic wave motion. 


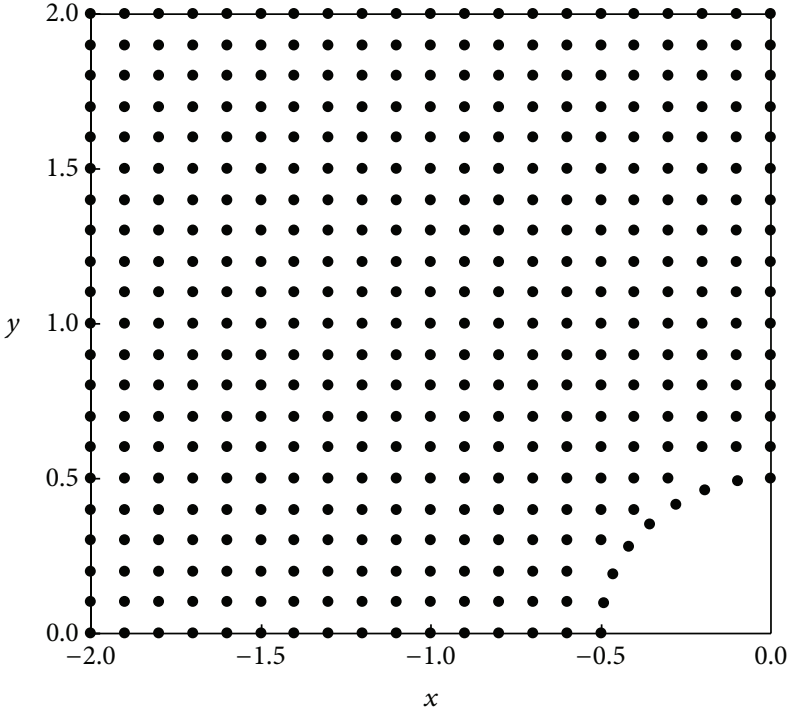

(a)

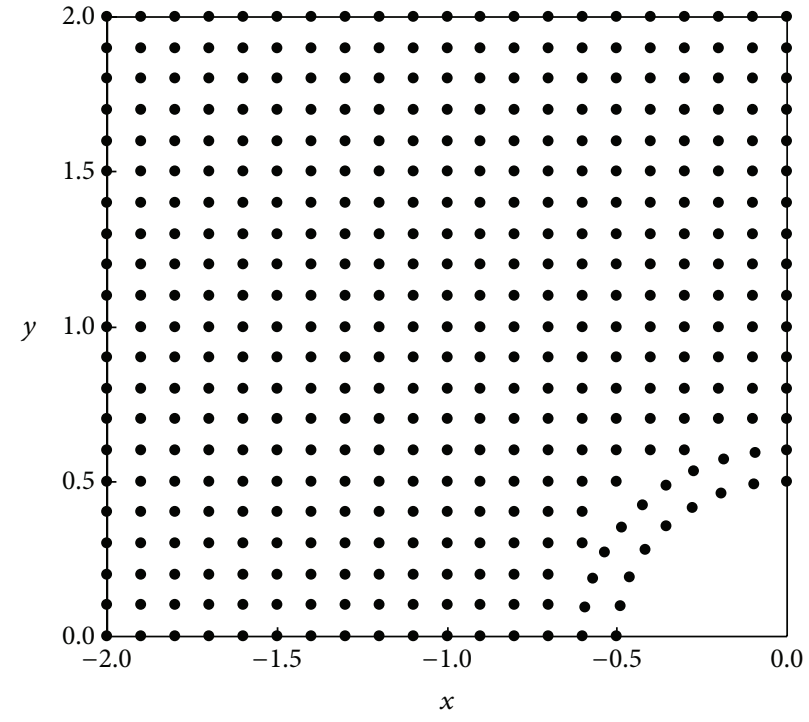

(b)

Figure 3: Two types of nodal arrangement for a potential uniform flow passing a circular cylinder.

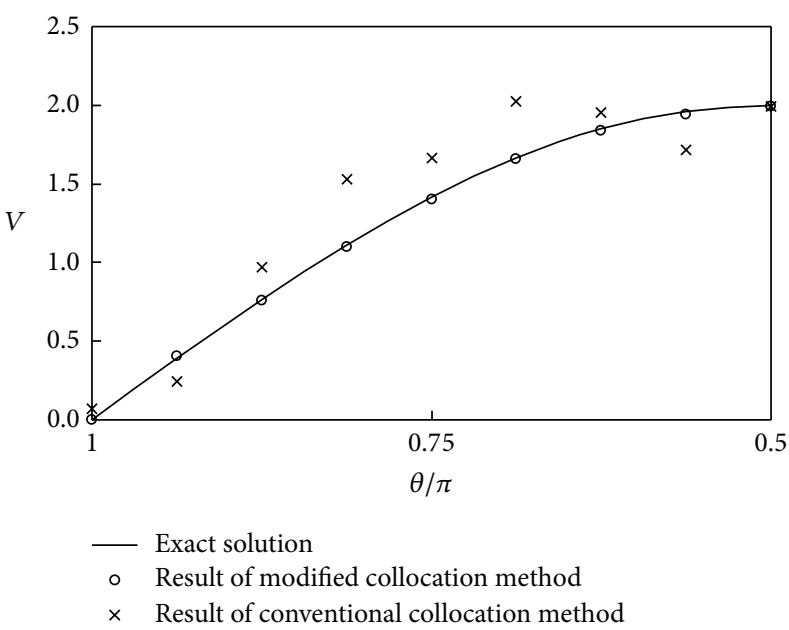

(a)

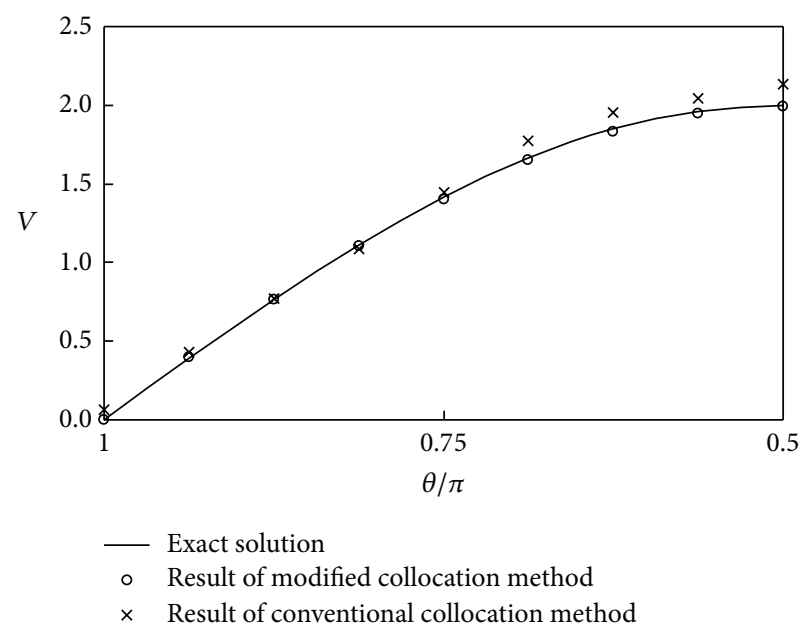

(b)

FIGURE 4: Computed flow speed profile along the body surface in the problem of a uniform flow passing a circular cylinder by two different nodal arrangements.

In the problem of monochromatic waves diffracted by a vertical cylinder in a constant water depth, shown as in Figure 8, the outer boundary has to be located far away from the cylinder and is given as the Sommerfeld [55] radiation condition of the scattered waves, $\phi^{S}$ :

$$
\lim _{\sqrt{r} \rightarrow \infty}\left(\frac{\partial \phi^{S}}{\partial r}-i k \phi^{S}\right)=0 .
$$

For linear progressive, monochromatic waves, the total velocity potential of wave motion equals the linear combination of incident wave and scattered waves, that is, $\phi=\phi^{I}+\phi^{S}$, and (31) can be implemented at an artificial open boundary far away from the scatter as

$$
\frac{\partial \phi}{\partial r}-i k \phi=\frac{\partial \phi^{I}}{\partial r}-i k \phi^{I}, \quad \text { at } r=r_{0} \gg 0
$$

where $r=\sqrt{x^{2}+y^{2}}$ and $\phi^{I}=a_{I} e^{i S}$ is the pseudo free surface velocity potential of incident wave in which $S$ denotes its phase function, and $a_{I}$ denotes wave amplitude. For waves propagating toward the $+x$ direction, the phase $S$ of 

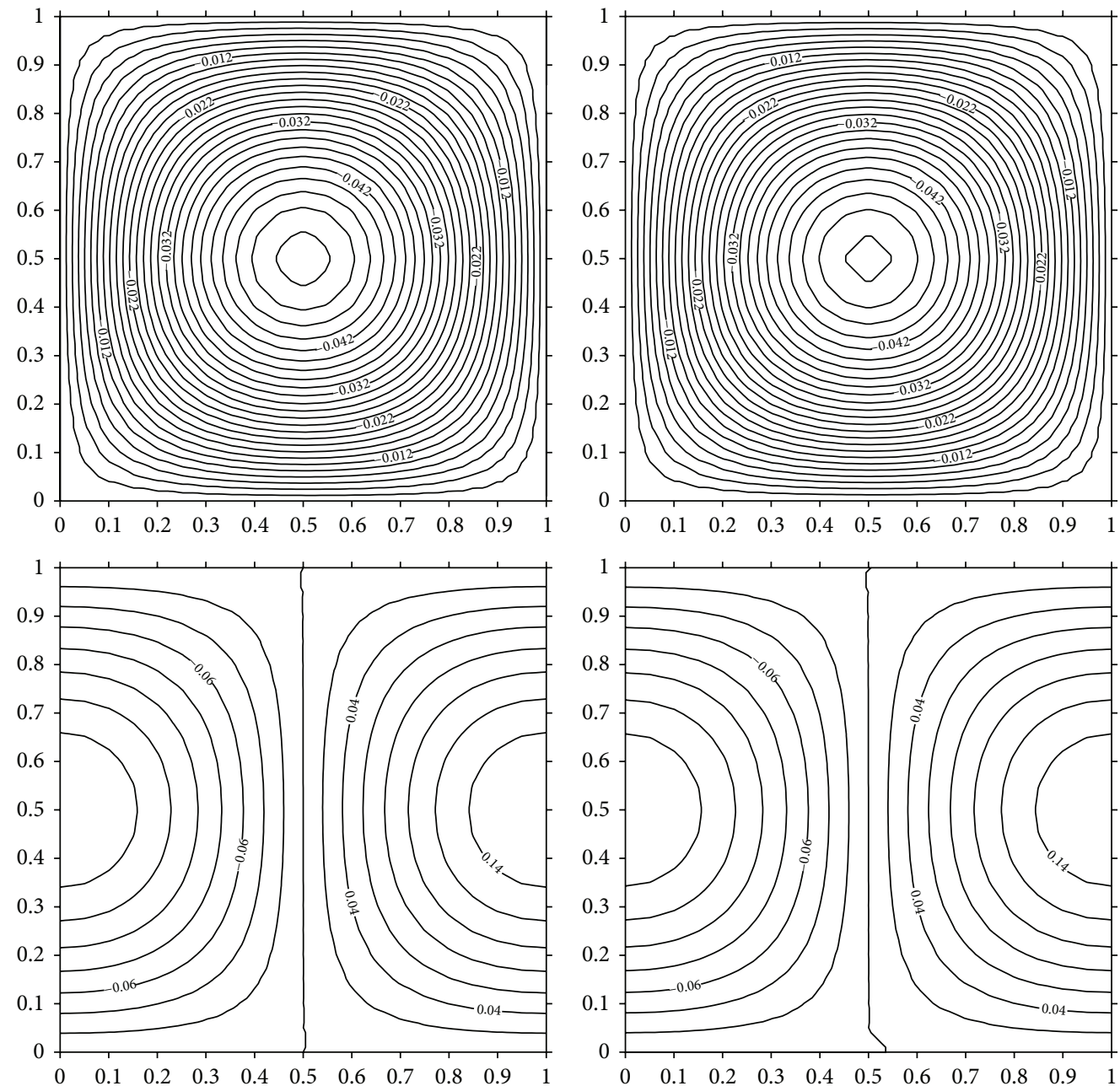

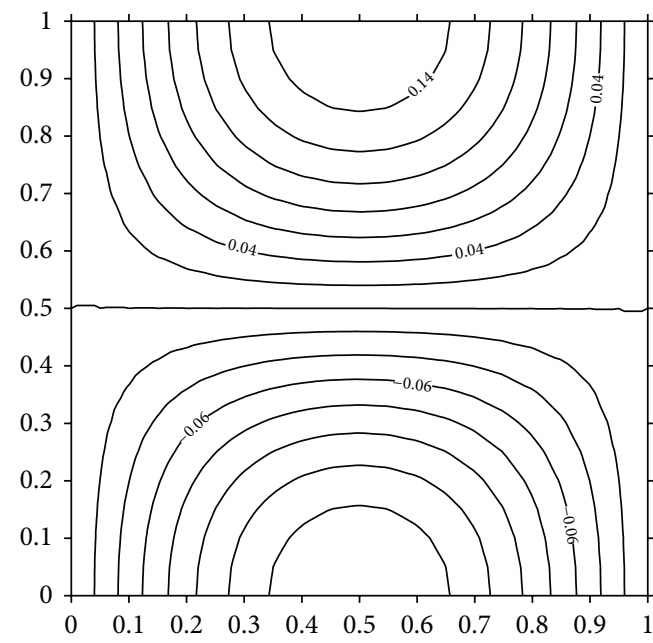

(a)

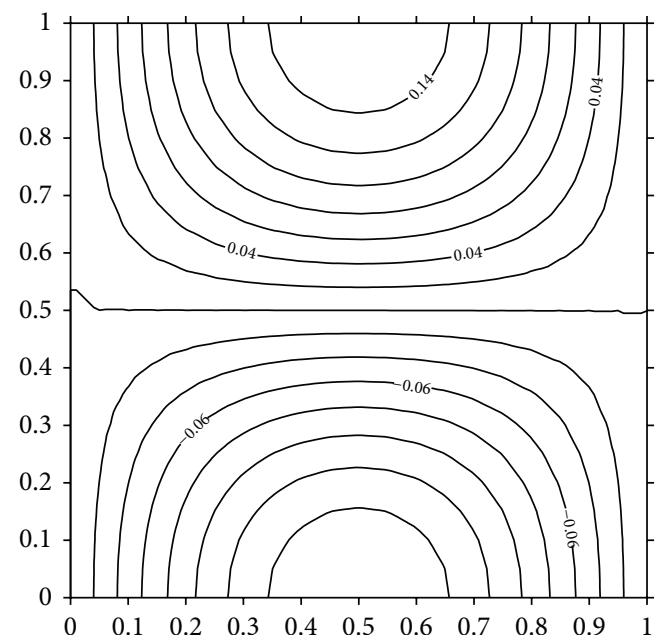

(b)

Figure 5: Comparisons of the numerical results (a) with the exact solutions (b) for the benchmark problem of Poisson equation. From top to bottom, they are the value of the solution and partial derivatives in $x$ - and $y$-direction, respectively. 


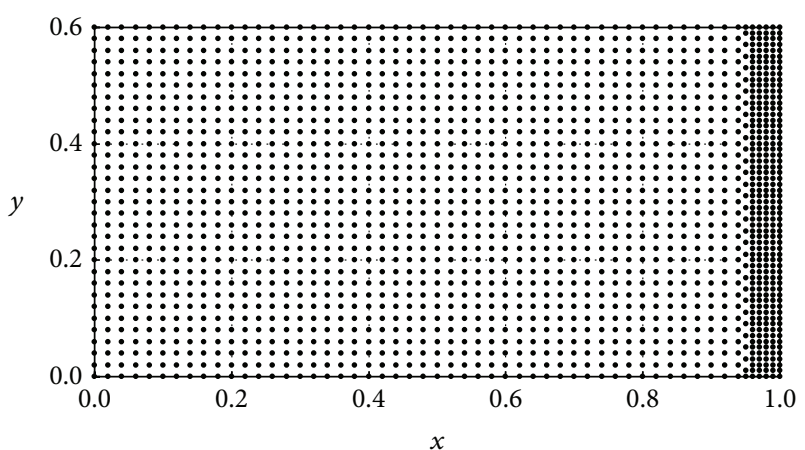

Figure 6: Nodal arrangement for the problem of the steady-state convection-diffusion equation.

the incoming wave is thus $i k x$. The no-flux boundary condition given at the cylinder surface is

$$
\frac{\partial \phi}{\partial r}=0, \quad \text { at } r=a,
$$

where $a$ is the radius of cylinder.

The analytical solution [56] of the problem governed by (29) subjected with the boundary condition given as (31)-(32) is

$$
\phi=a_{I} \sum_{n=0}^{\infty} \varepsilon_{n} i^{n}\left\{J_{n}(k r) H_{n}(k r) \times \frac{J_{n}^{\prime}(k a)}{H_{n}^{\prime}(k a)}\right\} \cos n\left(\theta-\theta_{I}\right),
$$

where

$$
\varepsilon= \begin{cases}1, & \text { if } n=0 \\ 2, & \text { if } n>0\end{cases}
$$

and $\theta_{I}$ is the incident angle of waves, and $J_{n}$ and $H_{n}$ are the Bessel and Hankel functions of the first kind of order $n$. The superscript of the symbol of prime indicates their derivatives, respectively. The radial and tangential velocities are obtained by the differentiation of the analytical solution with respect to $r$ and $\theta$, respectively:

$$
\begin{aligned}
U_{r}= & \frac{\partial \phi}{\partial r}=\frac{g a_{I}}{\omega} \\
& \times \sum_{n=0}^{\infty} \varepsilon_{n} i^{n-1}\left\{k J_{n}^{\prime}(k r)-k H_{n}^{\prime}(k r) \frac{k J_{n}^{\prime}(k a)}{k H^{\prime}(k a)}\right\} \\
& \times \cos n\left(\theta-\theta_{I}\right) \\
U_{\theta}= & \frac{1}{r} \frac{\partial \phi}{\partial \theta}=\frac{g a_{I}}{\omega r} \\
& \times \sum_{n=0}^{\infty} \varepsilon_{n} i^{n-1}\left\{-J_{n}(k r)+k H_{n}^{\prime}(k r) \frac{k J_{n}^{\prime}(k a)}{H_{n}^{\prime}(k a)}\right\} \\
& \times n \sin n\left(\theta-\theta_{I}\right) .
\end{aligned}
$$

A case of water depth of $10 \mathrm{~m}$ and incident waves with period $T=20 \mathrm{~s}$, which corresponds to wave number $k=$
$0.0308 \mathrm{~m}^{-1}$, is chosen for demonstration. The radius of the cylinder is chosen as $30 \mathrm{~m}$ while the open outer boundary is located at $r_{0}=295 \mathrm{~m}$. The nodal arrangement for the computation is shown in Figure 9. Around the first circle (i.e., the cylinder boundary), 30 nodes are placed. The number of nodes in each outer circle is increased by 6 while the radius of the circle is increased by $6.28 \mathrm{~m}$. Totally 43 circles (including cylinder boundary) are arranged so there are 6,708 nodes. Contours of numerical results of amplitude, phase, and magnitude of velocity within part of the computational domain are compared with analytical solutions as shown in Figures 10, 11, and 12. Numerical results of amplitude, phase, and magnitude of velocity around the cylinder are compared with the analytical solutions in Figure 13. Very good agreement in these comparisons implies that the MFPM is applicable not only to partial differential equation of real variables, but also to those of complex variables.

\section{Further Applications}

After the correctness and applicability of MFPM are verified in the previous section, applications of MFPM to several problems are further demonstrated in this section.

4.1. Numerical Generation of Orthogonal Grids. Though meshless numerical methods are becoming more and more popular in recent decades, grid-based numerical methods, which have been fully developed, are still indispensable. Transformation of coordinates from Cartesian system $(x-y$ coordinate) to a curvilinear system ( $\xi-\eta$ coordinate) plays an important role in the numerical simulations using grid-based methods. The most desirable transformation may be the one that could generate orthogonal grid lines on the boundaries as well as inside the domain, so the discretization of the governing equation is simplified and thus the discretization error in solving the governing equation could be minimized [57]. The orthogonal coordinate transformation can be accomplished by solving two pairs of Laplace equations and subjected to the Cauchy-Riemann boundary conditions [58].

One application of MFPM is to employ it to solve the Laplace equations of numerically generate 2-D orthogonal grids. Based on Cauchy-Riemann conditions, the orthogonal transformation from the physical domain ( $x-y$ coordinate) to the computational domain $(\xi-\eta$ coordinate) can be reduced to a problem governed by two Laplace equations:

$$
\begin{aligned}
& \frac{\partial^{2} \xi}{\partial x^{2}}+\frac{\partial^{2} \xi}{\partial y^{2}}=0 \\
& \frac{\partial^{2} \eta}{\partial x^{2}}+\frac{\partial^{2} \eta}{\partial y^{2}}=0
\end{aligned}
$$

with the boundary conditions

$$
\begin{array}{ll}
\vec{n} \cdot \nabla \eta=0 & \text { on } \xi=0 \text { and } \xi=\xi_{0} \\
\vec{n} \cdot \nabla \xi=0 & \text { on } \eta=0 \text { and } \eta=\eta_{0}
\end{array}
$$




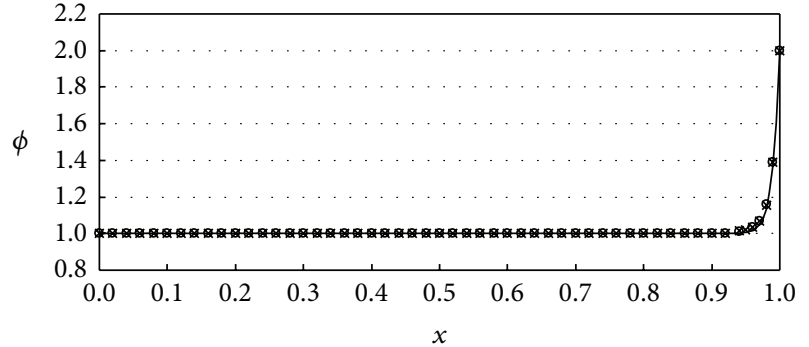

- Exact solution

- Numerical solution at $y=0.3$

$\times \quad$ Numerical solution at $y=0$

(a)

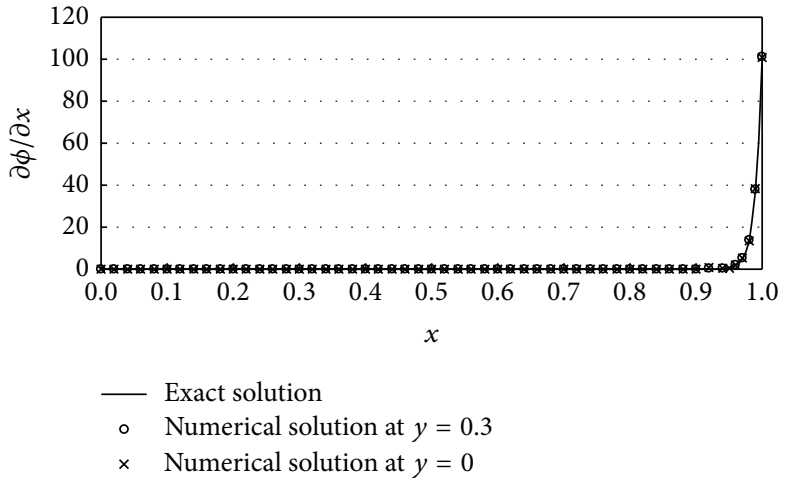

(b)

FIGURE 7: Results of problem of the steady-state convection-diffusion equation.

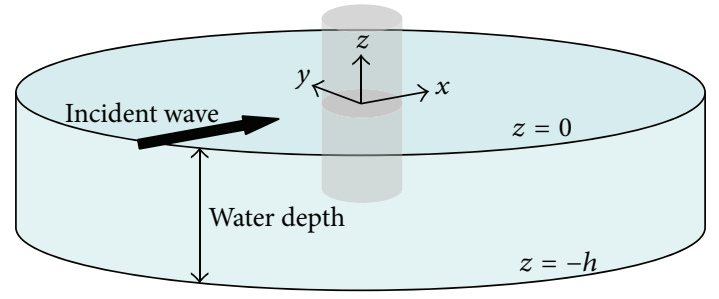

FIGURE 8: A sketch of progressive monochromatic waves scattered by a vertical cylinder in a constant water depth.

where $\vec{n}$ is the outward normal vector of the boundaries. After (37a) and (37b) are solved separately, the obtained $x-y$ to $\xi-\eta$ one-to-one corresponding relationship can be used as the boundary conditions for seeking the inverse transformation which is also governed by two Laplace equations:

$$
\begin{aligned}
& \frac{\partial^{2} x}{\partial \xi^{2}}+\frac{\partial^{2} x}{\partial \eta^{2}}=0 \\
& \frac{\partial^{2} y}{\partial \xi^{2}}+\frac{\partial^{2} y}{\partial \eta^{2}}=0
\end{aligned}
$$

The relations between the partial derivatives are listed below:

$$
\begin{aligned}
& \frac{\partial \xi}{\partial x}=\frac{1}{J} \frac{\partial y}{\partial \eta} \\
& \frac{\partial \xi}{\partial y}=\frac{-1}{J} \frac{\partial x}{\partial \eta} \\
& \frac{\partial \eta}{\partial x}=\frac{-1}{J} \frac{\partial y}{\partial \xi} \\
& \frac{\partial \eta}{\partial y}=\frac{1}{J} \frac{\partial x}{\partial \xi}
\end{aligned}
$$

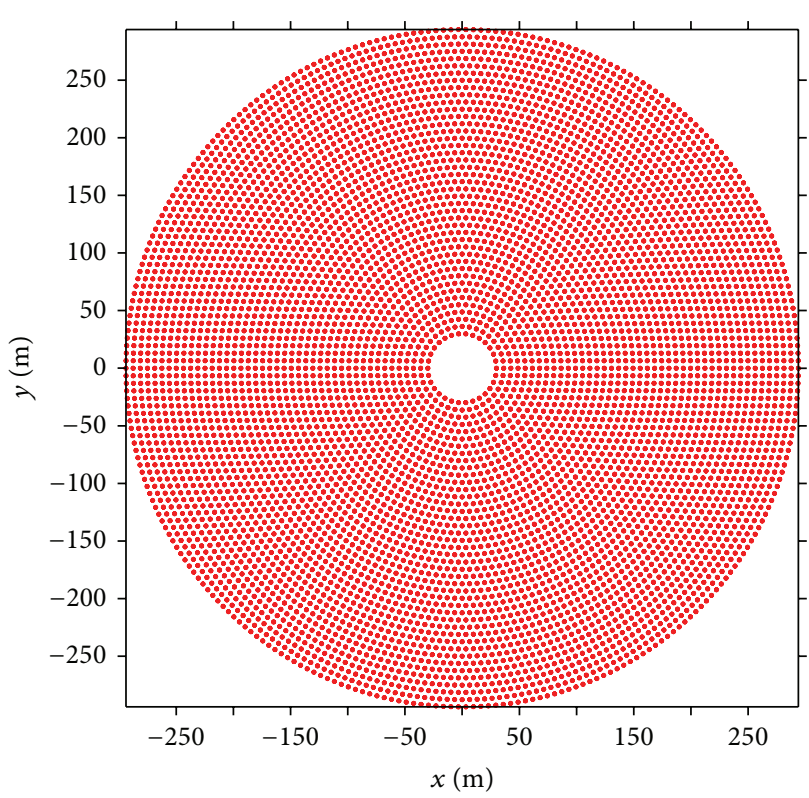

FIGURE 9: Nodal arrangement for MFPM computations of wave scattering.

where $J$ is the Jacobian and is defined as

$$
J=\frac{\partial x}{\partial \xi} \frac{\partial y}{\partial \eta}-\frac{\partial x}{\partial \eta} \frac{\partial y}{\partial \xi}
$$

A sketch of the transformation is illustrated in Figure 14. A detailed derivation of these equations could be found in [58].

Conventionally the four corners of the irregular domain have to be right angles and the irregular domain is denominated as a "hyper-rectangle" [59-62], which consists of four right corners and four smooth curves. A method of conformal mapping in the complex-value domain is integrated to transform any irregular geometry into a hyper-rectangle has been developed in [59-62], except the case which contains interior corners of zero-degree angle. The grid generation 


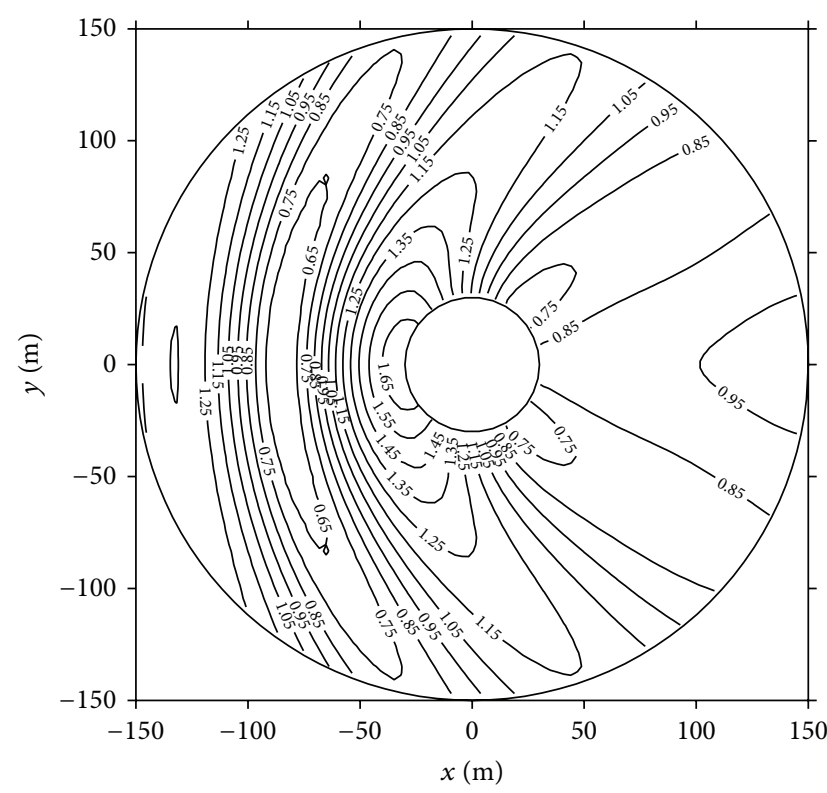

(a)

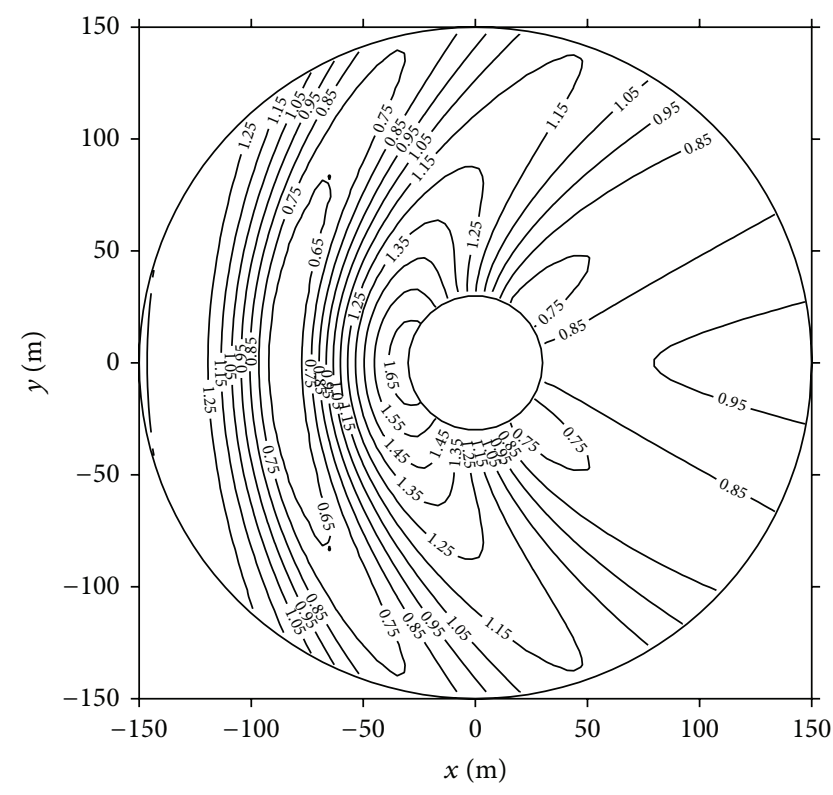

(b)

FIGURE 10: Contours of equal-amplitude of waves scattered by a cylinder in constant water depth, $T=20$ s. (a) MFPM results; (b) analytical solutions.

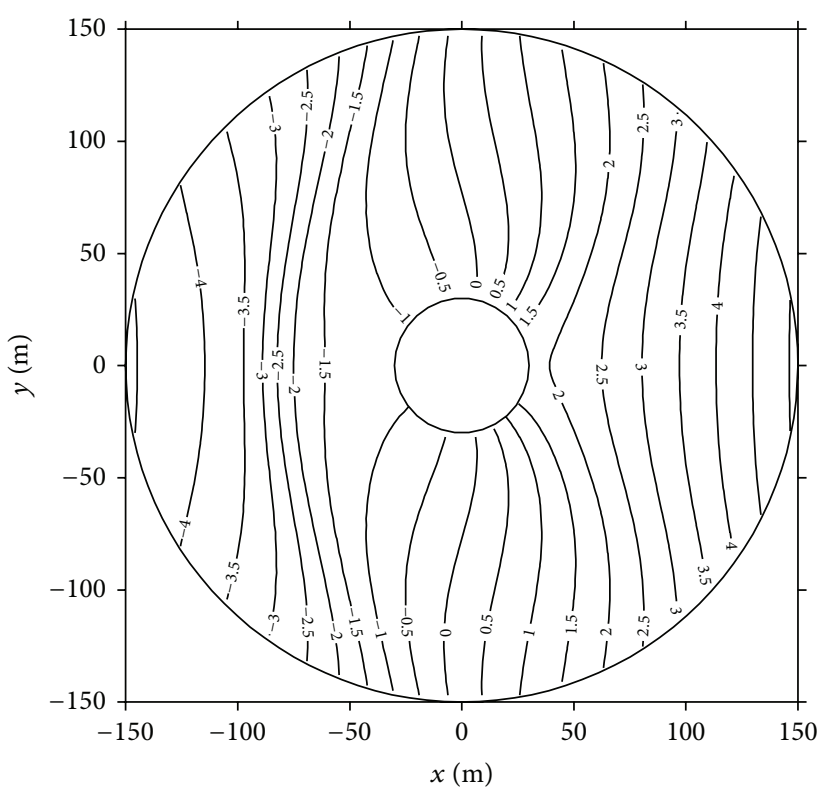

(a)

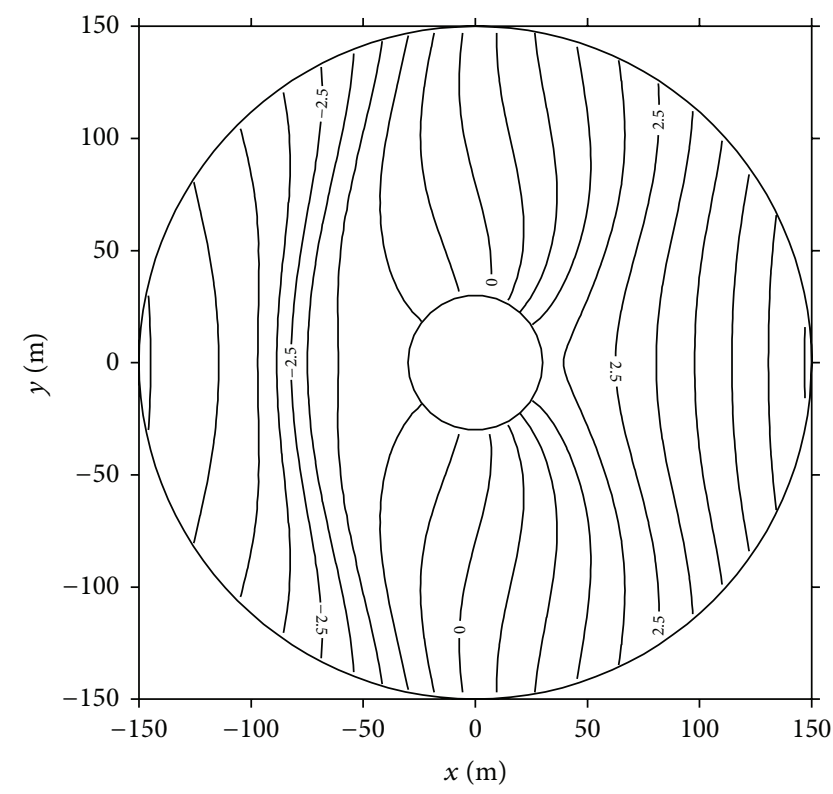

(b)

Figure 11: Contours of equal-phase of waves scattered by a cylinder on constant water depth, $T=20 \mathrm{~s}$. (a) MFPM results; (b) analytical solutions.

in a region shown in Figure 15 was a bottleneck in previous works [59-62], because of a local complex transformation failed at points with zero-degree internal angle. The orthogonal grid generation in this irregular region with zero-degree internal corners is presented here for completeness. The values of $\xi_{0}$ and $\eta_{0}$ are all set to be 1 because of the symmetry.
For forward transformation from $x-y$ to $\xi-\eta$, the collocation points inside the domain are regularly distributed with a $45-$ degree inclination, as shown in Figure 16. The spacing of collocation points on the boundary is $1 / 80$ of the arc length. Collocation points are selected in the way that the nearest distance between any two collocation points is greater than 


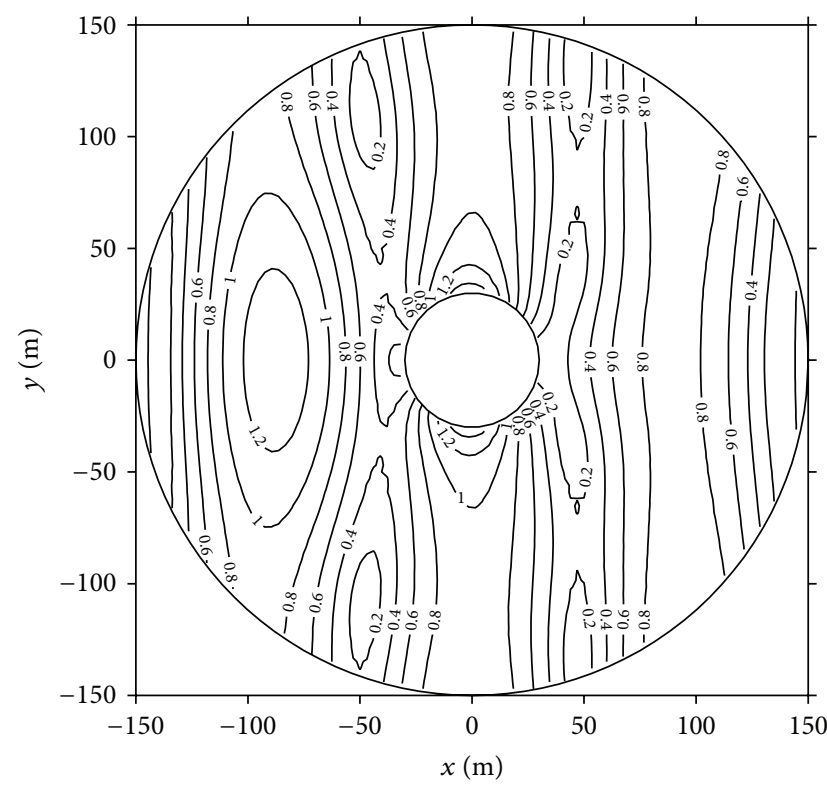

(a)

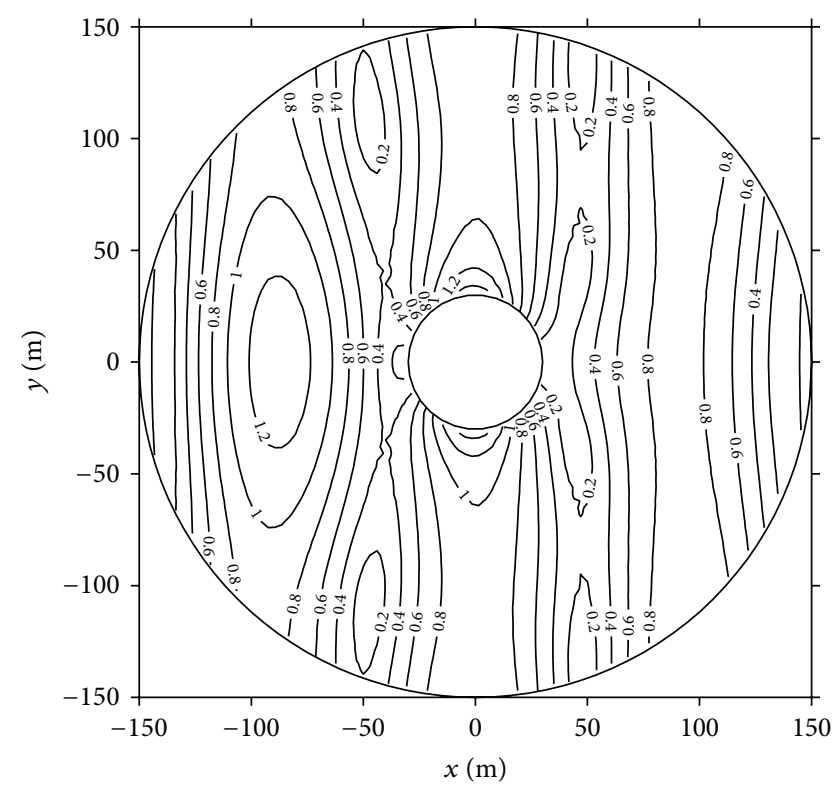

(b)

Figure 12: Contours of magnitude of velocity of waves scattered by a cylinder on constant water depth, $T=20$ s. (a) MFPM results; (b) analytical solutions.

0.007. So some collocation points around the zero-degree internal corners are skipped.

There is no exact solution for this problem, but one can still verify the correctness of the transformation by checking the orthogonality. Because the orthogonality is guaranteed mathematically, the following relative error criteria are used to show the correctness of the results:

$$
\begin{array}{r}
\operatorname{ARE}_{O}=\sqrt{\frac{\sum_{j=1}^{N}(\nabla \xi \cdot \nabla \eta)_{\vec{x}=\vec{x}_{j}}^{2}}{\sum_{j=1}^{N}\left(|\nabla \xi|^{2}|\nabla \eta|^{2}\right)_{\vec{x}=\vec{x}_{j}}}} \\
\mathrm{MRE}_{O}=\sqrt{\frac{\max \left\{(\nabla \xi \cdot \nabla \eta)_{\vec{x}=\vec{x}_{j}}^{2}, j=1 \sim N\right\}}{\left(\frac{1}{N} \sum_{j=1}^{N}\left(|\nabla \xi|^{2}|\nabla \eta|^{2}\right)_{\vec{x}=\vec{x}_{j}}\right)}} .
\end{array}
$$

The subscript " $O$ " denotes "orthogonal." The smaller the values of these two relative error criteria are, the higher the correctness of the numerical results will be. MFPM numerical result shows that the averaged relative error and maximum relative error calculated by the collocation points are $7.60 \times$ $10^{-5}$ and $7.53 \times 10^{-4}$, respectively.

Choosing $\Delta \xi=\Delta \eta=0.05$, generated grid lines are plotted in Figure 17. Each line of one set is observed very well perpendicular to all the lines of the other set. To illustrate more details of the grid lines around a zero-degree internal corner, grid lines with $\Delta \xi=\Delta \eta=0.01$ are plotted in Figure 18. The density of the grid nodes is still very coarse near the corner. The point $(\xi, \eta)=(0.99,0.99)$ is far away from the corner, compared with the distances from other neighboring $\xi-\eta$ grid nodes to it. This implies that the scaling factors $\partial \xi / \partial x, \partial \xi / \partial y, \partial \eta / \partial x$, and $\partial \eta / \partial y$ approach to zero at the corners. One could find more details of this application and other applications of MFPM to such kind of problems in [47].

\subsection{Frequency-Domain Water Wave Problems}

4.2.1. Combined Refraction and Diffraction of Water Waves. Various numerical methods have been developed to study the combined water-wave refraction and diffraction problems in the last decade. Notably, Tsay and Liu [63] based on the linear long-wave theory developed a hybrid finite element model, which can be used for studying harbor oscillations and calculating wave forces and moments on offshore structures. Following the same idea of using a hybrid method, Yue et al. [64] developed a three-dimensional finite element model which is applicable for a wider range of wave lengths. However, the three-dimensional model requires huge storage and computational time when the incident wave length becomes short.

Alternatively, the mild-slope equation derived by Berkhoff [65] and Smith and Sprinks [66] has gained popularity as a base for studying combined refraction and diffraction of water waves, since it reduces a three-dimensional problem to a two-dimensional one. Furthermore, this equation is also applicable to a wide range of wave frequencies. Of course, the drawbacks for using the mild-slope equation are that water depth must be assumed to be slowly varying in space and the evanescent modes are ignored. Nevertheless, Bettess and Zienkiewicz [67], Houston [68, 69], and Tsay and Liu [63] developed by hybrid methods based on the mild-slope equation. Excellent results were obtained for problems concerning wave scattering by islands as well 


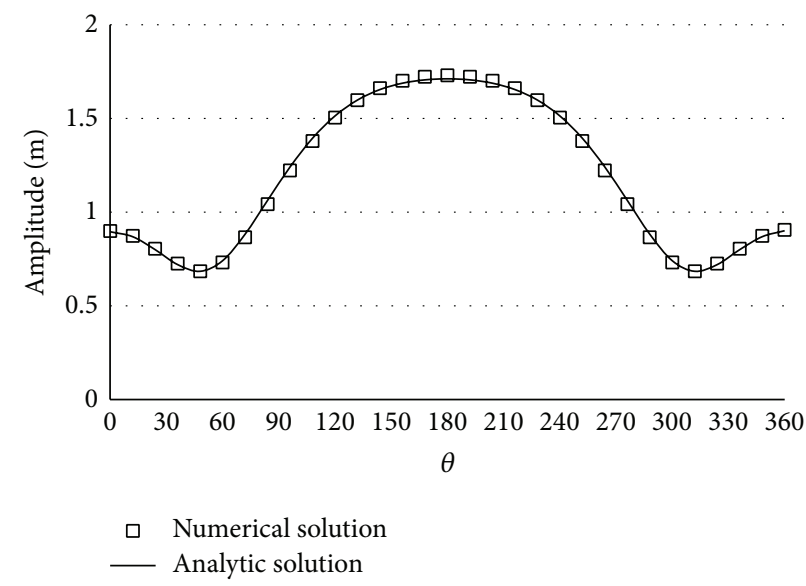

(a)

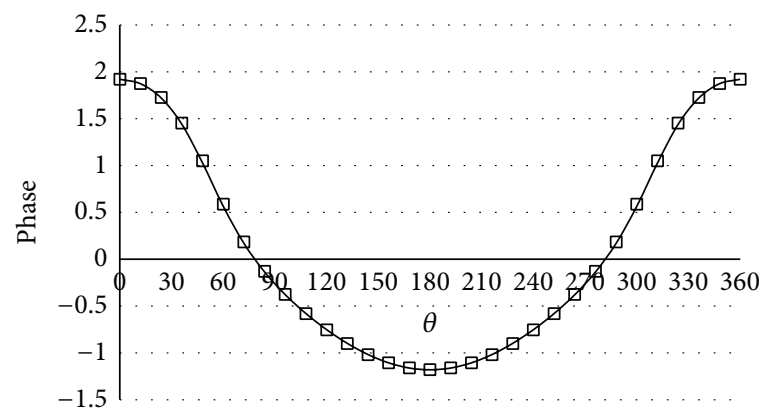

- Numerical solution

- Analytic solution

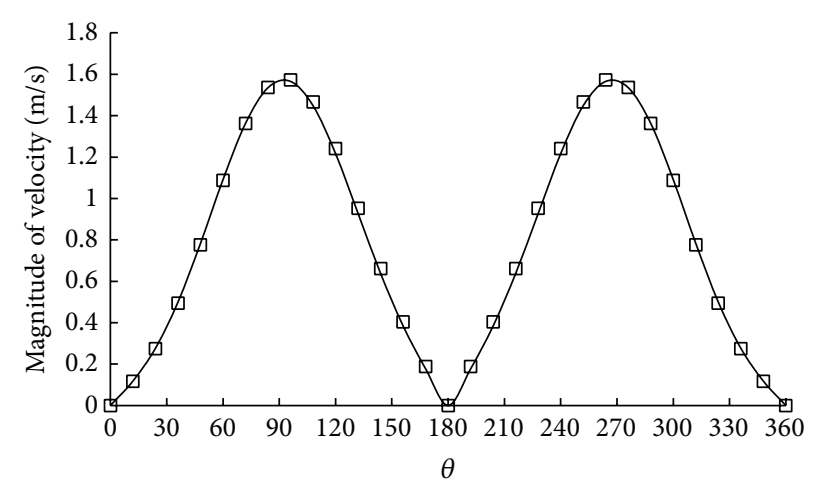

口 Numerical solution

- Analytic solution

(c)

FIGURE 13: Comparisons of MFPM results and analytical solutions of amplitude, phase, and magnitude of velocity around at $r=a$.

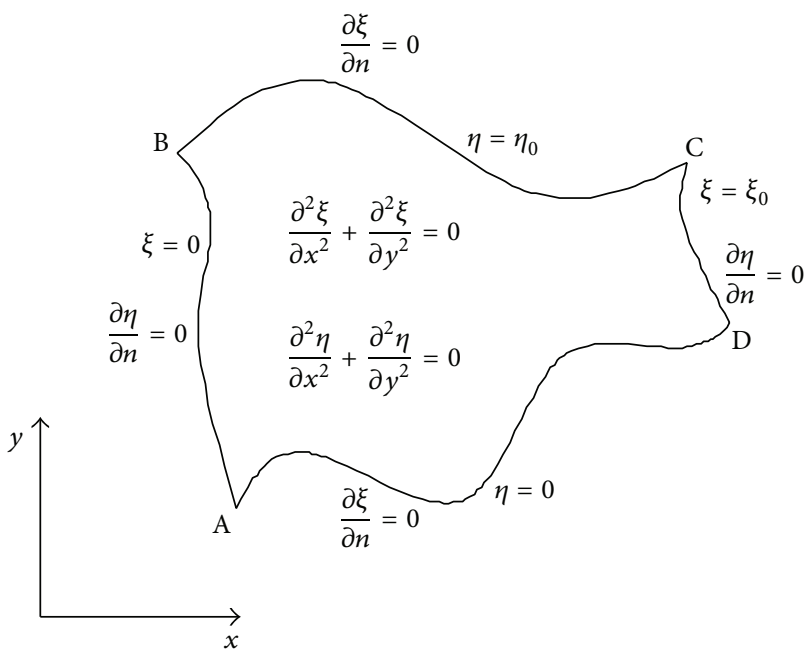

(a)

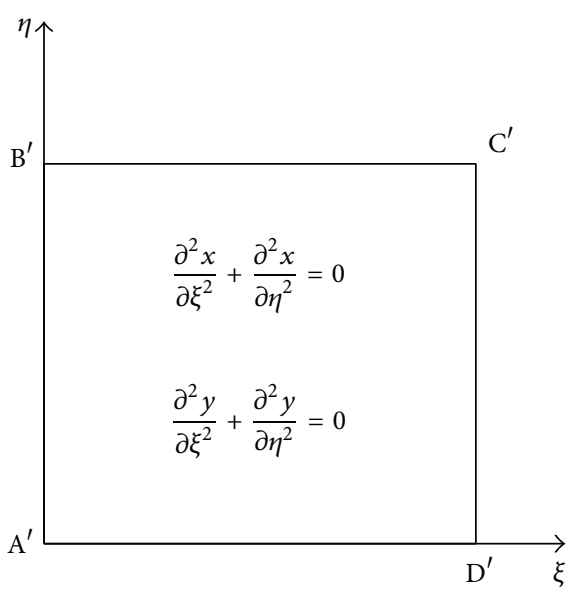

(b)

FIGURE 14: Sketch of a transformation between two orthogonal coordinates from the physical domain to the transformed domain. 


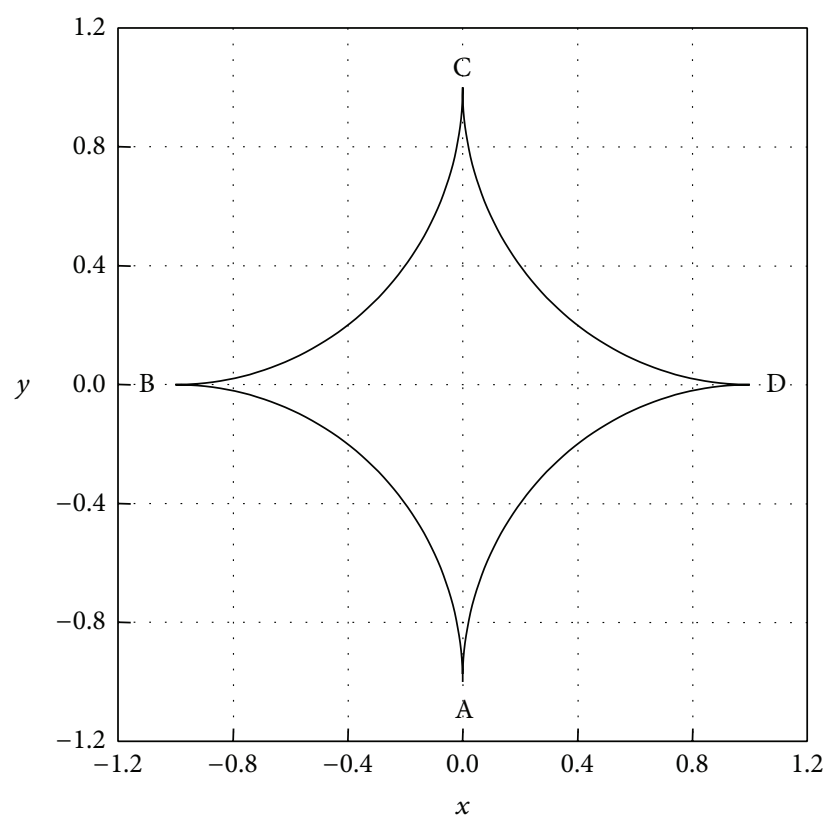

FIGURE 15: The domain of an irregular area with zero-degree internal corners.

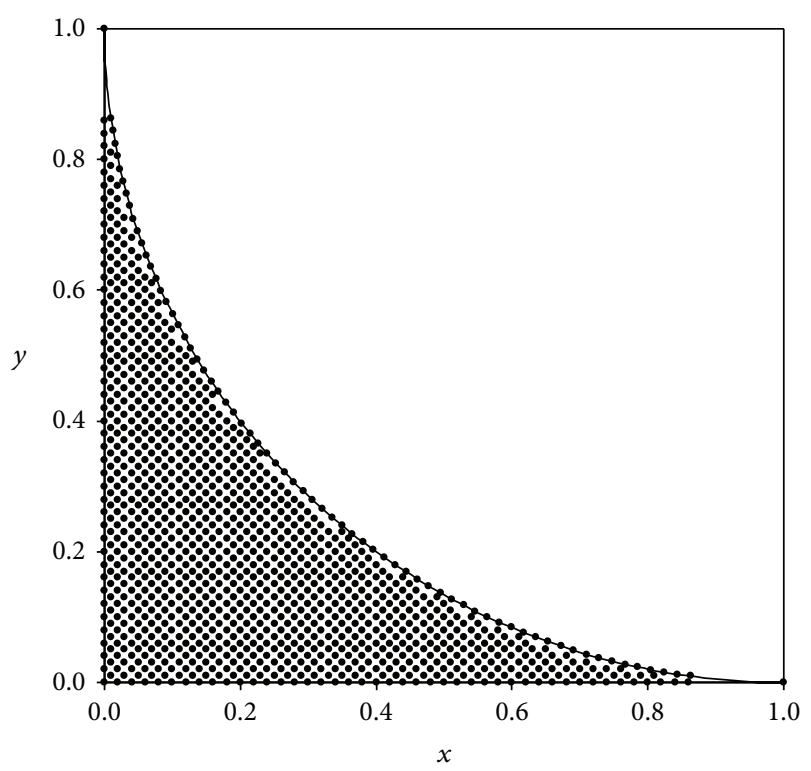

FIGURE 16: Arrangement of the collocation points in the up-right quarter of the irregular area with corners with zero-degree angle.

as harbor oscillations. In Bettess and Zienkiewicz's [67] work, infinite elements were used in the region away from the scatters. Houston $[68,69]$ showed that his model can generate accurate solutions by using very fine elements. Neither model can, however, calculate wave forces on a semi-submerged offshore structure. Tsay and Liu [63] used a hybrid finite element model originally developed by Chen and Mei [70] for shallow water wave problems. The physical domain is divided into two regions as a finite inner region of variable depth and infinite outer region with constant or

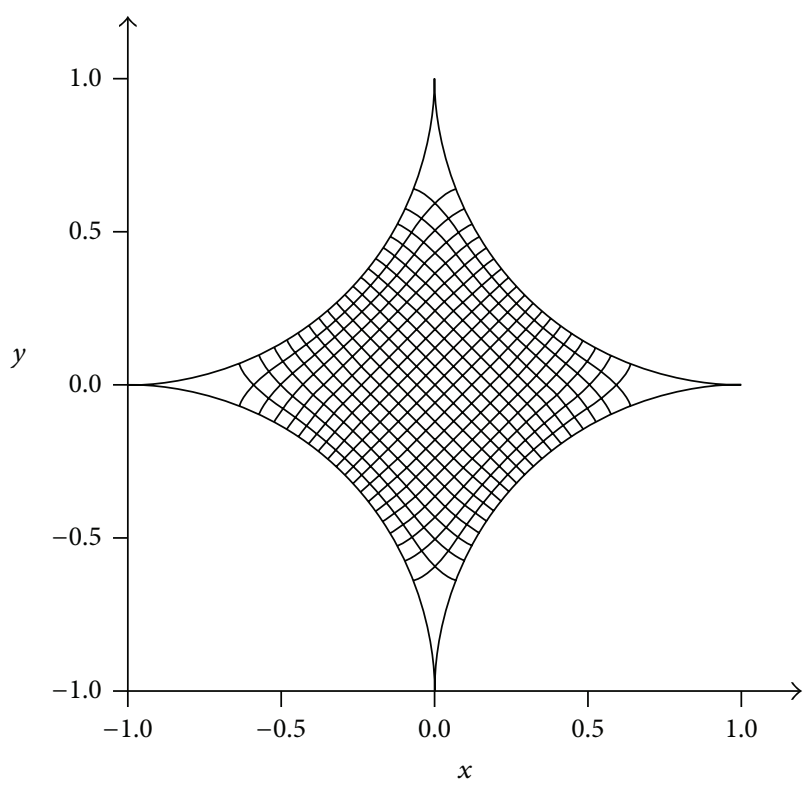

FIGURE 17: Orthogonal grid lines in an irregular area with internal corners of zero-degree angle, $\Delta \xi=\xi_{0} / 20, \Delta \eta=\eta_{0} / 20, \xi_{0}=1$, and $\eta_{0}=1$.

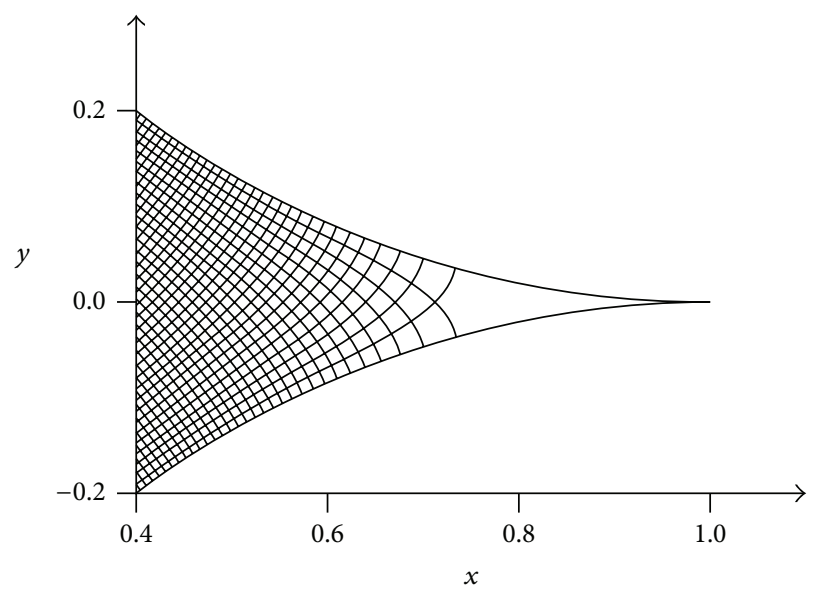

FIGURE 18: Orthogonal grid lines around a zero-degree internal corner, $\Delta \xi=\xi_{0} / 100, \Delta \eta=\eta_{0} / 100, \xi_{0}=1$, and $\eta_{0}=1$.

deep water depth. The infinite outer region is represented by analytical solutions with undetermined coefficients which satisfy the governing equation and the radiation condition on the outer boundary. Conventional discretization of finite element is used in the variable depth region.

Following the procedures that Berkhoff [65] used, the mild-slope equation for water waves could be briefly shown as

$$
\nabla \cdot\left(C C_{g} \nabla \eta\right)+k^{2} C C_{g} \eta=0
$$

where $C=\omega / k$ and $C_{g}=(1 / 2)(1+2 k h / \sinh 2 k h) C$ represent wave celerity and group celerity, respectively; $k$ represents the wave number. In case the water depth is a constant, (43) can be reduced to (29). 

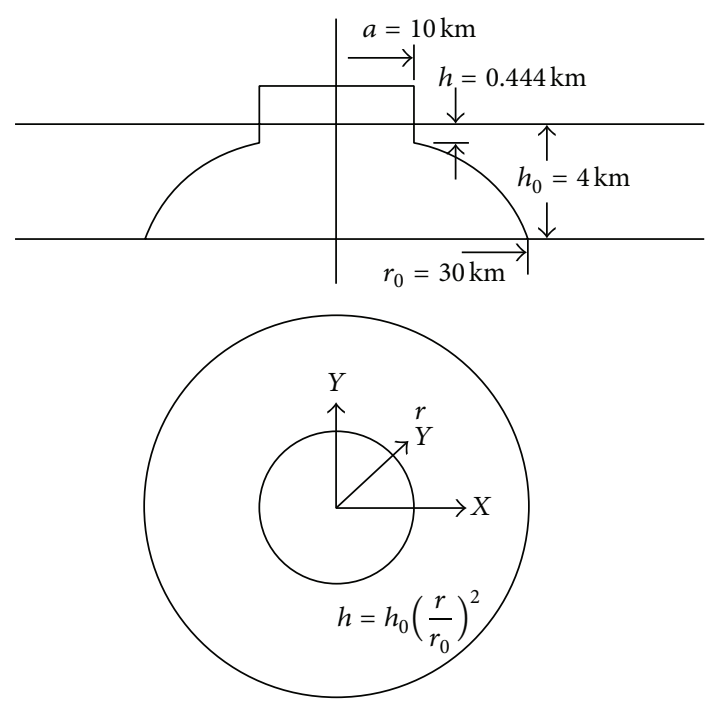

FIGURE 19: Definition of a circular island on a paraboloidal shoal.

Generally, the treatment of the boundary far from the cylinder is the same as in (32). When there are some manmade structures such as breakwaters and wharfs in harbors, a no-fluxing boundary at such solid boundaries is required and can be expressed as

$$
\frac{\partial \eta}{\partial n}=0
$$

In this section, the MFPM is implemented for the solution of the mild-slope equation for combined refraction and diffraction when monochromatic waves encountered with scatters, due to change of water depth or artificial structures. In present numerical model, the velocity of fluid particles at the grid points can be found easily as part of the coefficients resulted from the coefficients of collocation at each computational node. To verify present numerical model, analytical solutions of a benchmark problem in shallow water waves scattered by a circular island on a paraboloidal shoal by Homma [71] have been applied. It should be noted that the unknown variables of the numerical and analytic solutions are complex numbers.

As shown in Figure 19, the depth between the shoreline and outer boundary of the shoal is described as

$$
h=h_{0}\left(\frac{r}{r_{0}}\right)^{2}, \quad a \leq r \leq r_{0},
$$

where $r$ is the distance measured from the center of the island, $a$ is radius of the circular island, $r_{0}$ is the radius of paraboloidal shoal, and $h_{0}$ is the constant depth outside the paraboloidal shoal.

Analytical solutions of propagating shallow water wave responses around this circular island for $a \leq r \leq r_{0}$, derived by Homma [71], are presented as the following for completeness:

$$
\begin{aligned}
\eta= & a_{0} \sum_{n=0}^{\infty} k \varepsilon_{n} i^{2 n}\left[J_{n}^{\prime}\left(k r_{0}\right) H_{n}\left(k r_{0}\right)-J_{n}\left(k r_{0}\right) H_{n}^{\prime}\left(l r_{0}\right)\right] \\
& \times\left(\frac{\alpha_{n}+1}{\alpha_{n}-1} a^{-2 \alpha_{n}} r^{\alpha_{n}-1}+r^{-\alpha_{n}-1}\right) \cos n \theta \\
+ & {\left[\left(\alpha_{n}+1\right)\left(a^{-2 \alpha_{n}} r_{0}^{\alpha_{n}-2}-r_{0}^{-\alpha_{n}-2}\right) H_{n}\left(k r_{0}\right)\right.} \\
& \left.\quad-k H_{n}^{\prime}\left(k r_{0}\right)\left(\frac{\alpha_{n}+1}{\alpha_{n}-1}\right) a^{-2 \alpha_{n}} r_{0}^{\alpha_{n}-1}+r_{0}^{-\alpha_{n}-1}\right] .
\end{aligned}
$$

$\theta_{I}$ is the incident angle of propagating waves and $J_{n}$ and $H_{n}$ are the Bessel and Hankel functions of the first kind of order $n$, as in the fourth benchmark problem. The superscript of the symbol of prime indicates their derivatives, respectively.

Differentiating (47) with respect to radial $(r)$ and angular $(\theta)$ variables, respectively, the analytical solutions of radial and tangential velocities in the inner region of $a \leq r \leq r_{0}$ can be obtained analytically with any difficulty.

A case for a circular island with radius, $a=10 \mathrm{~km}$, as shown in Figure 19, is considered. The water depth is constant, $h_{0}=4 \mathrm{~km}$, at distance greater than $r_{0}=30 \mathrm{~km}$. The amplitude and period of incident wave are $1 \mathrm{~m}$ and $720 \mathrm{~s}$, respectively. The radius of the local radiation boundary is $275 \mathrm{~km}$ which is about 2 times of wave length from the paraboloidal shoal boundary. 120 nodes are arranged at the circumference of the circular island. For the next circle, the spaces of radial and tangential directions of nodes increased by $5 \%$. After 55 circles of nodes, there are 4 circles increased by 6 more nodes for each one. In the case there are totally 9,910 nodes and 59 circles arranged. The numerical results of contours of amplitude, phase, and magnitude of velocity are compared with analytical solutions as shown in Figures 20, 21, and 22. Comparisons of numerical of results of amplitude, phase, and magnitude of velocity at $t=0$ with the analytical solutions around the margin of cylinder are shown in Figure 23. Good agreements in these comparisons imply the applicability of MFPM to complex variable problems and simulate water-wave scattering problems. It should be noted that the velocity components in $x$ - and $y$-direction, respectively, are part of the solutions in the coefficients of local polynomial collocations. Therefore, present MFPM is more efficient than other numerical methods using linear shape functions.

4.2.2. Harbor Resonance. Although present meshless methods have advantages over element- or mesh-based numerical methods to avoid tedious preprocessing of the mesh connectivity information in most the numerical computations, there are problems in engineering and science consisting of different regions in its physical behaviors divided by a very thin barrier or crack, such as a breakwater in water-wave diffraction, a crack in thin plates, and a cutoff in groundwater 


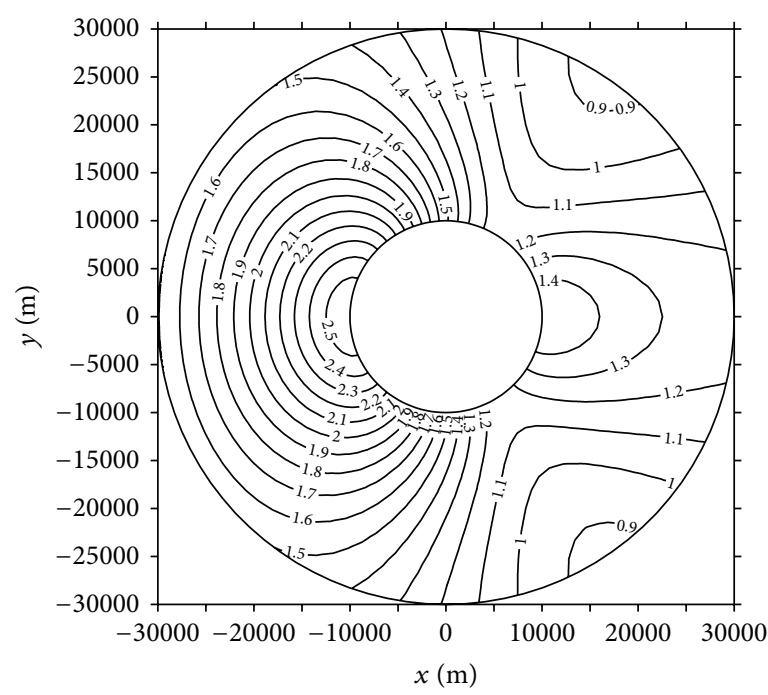

(a)

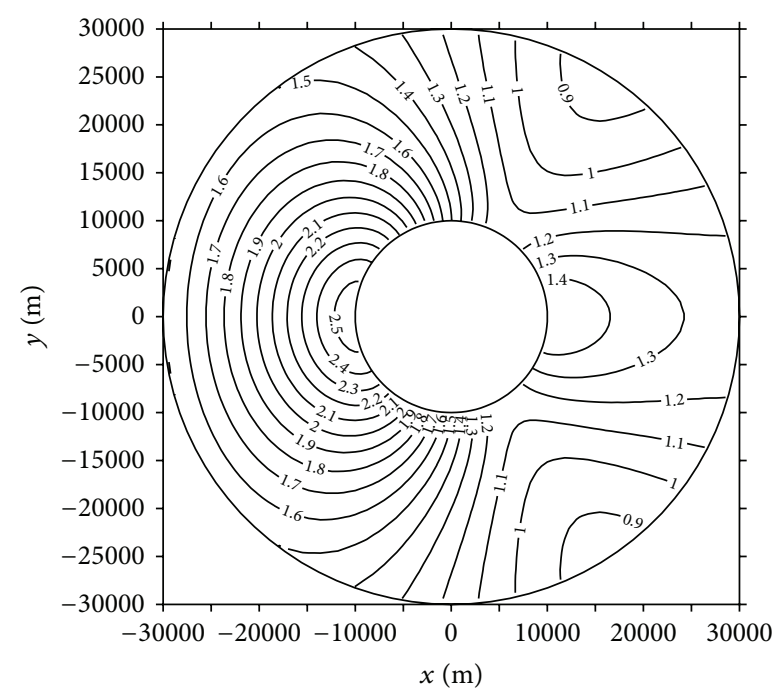

(b)

Figure 20: Contours of wave amplitude lines around a circular island on a paraboloidal shoal, $T=720$ s. (a) Numerical result; (b) analytic results.

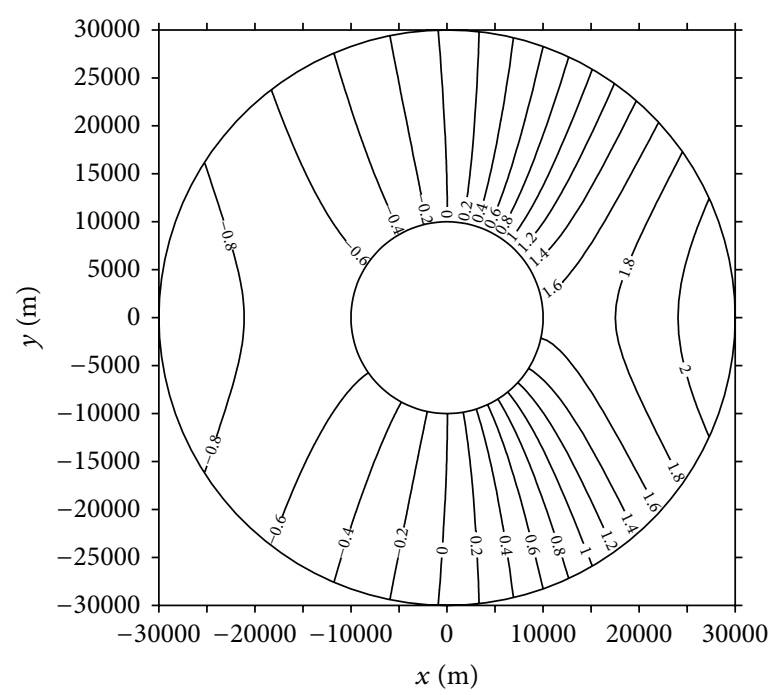

(a)

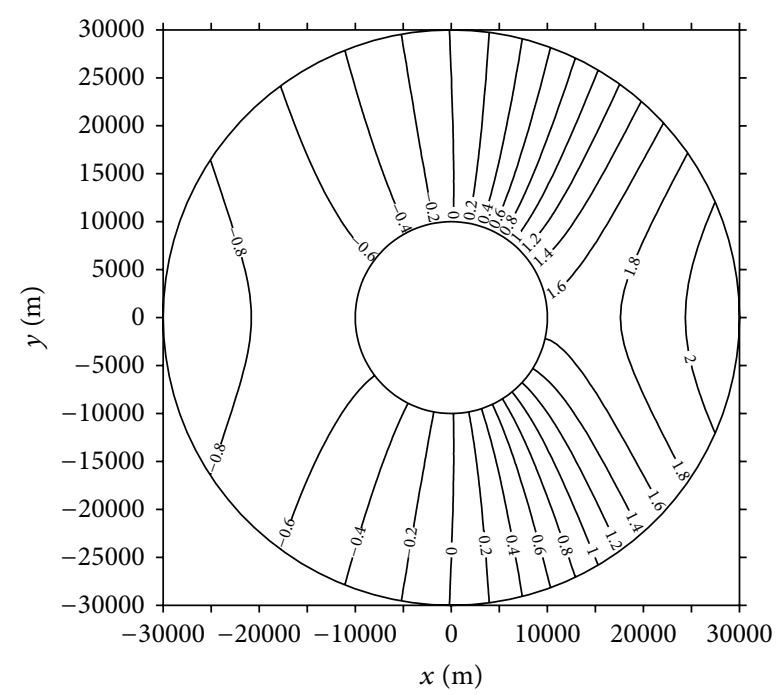

(b)

Figure 21: Contours of wave phase lines around a circular island on a paraboloidal shoal, $T=720 \mathrm{~s}$, at $t=0$. (a) Numerical results; (b) analytic results.

seepage. In present MFPM, the local collocation is performed by searching some closest nodes near the base point. When physical behaviors in separated regions by a thin barrier, it requires to identify the nodes searched by relative distance are not from the different regions with different physical behaviors. Therefore, it is required to divide the whole domain into subdomains of different physical behaviors. It is referred to as regional connectivity to complement the searching procedures of the local nodes for appropriate collocations [49]. In this section, applications of present MFPM will be demonstrated in coastal engineering problem for harbor resonance with breakwaters.
To study resonance phenomena in a harbor basin, Lee [72] first developed deep basin resonance theory (arbitraryshape harbor theory) and used separation of variables method to obtain the basin resonance for a circular harbor and a narrow channel. Compared with experimental measurements, the theoretical results of the narrow channel showed good agreement. Many numerical methods have been developed based on different linear, small-amplitude wave theory and obtained very good results $[63,65-67,70$, 73].

However, there exist two drawbacks of these kinds of numerical models. First, a tedious preprocessing procedure is 


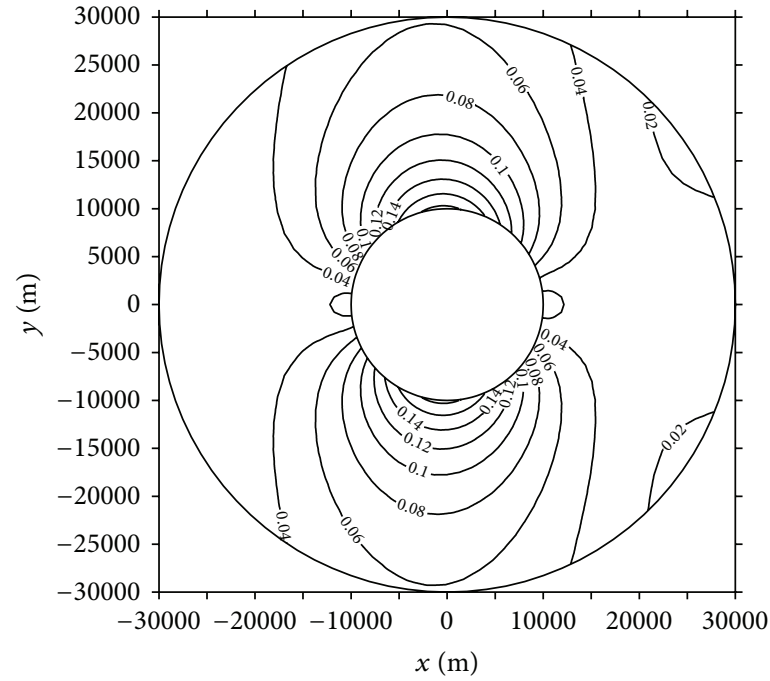

(a)

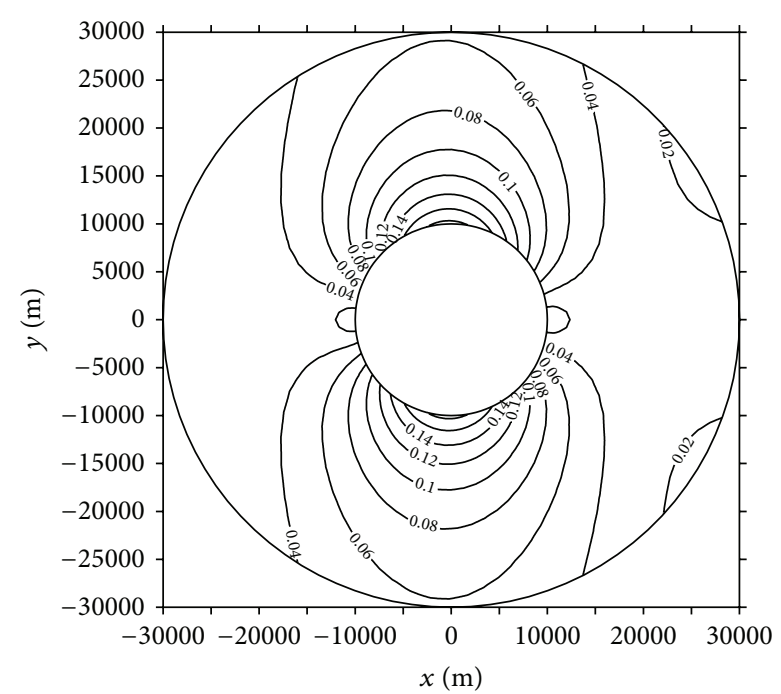

(b)

FIGURE 22: Contours of magnitude of velocity around a circular island on a paraboloidal shoal, $T=720 \mathrm{~s}, t=0$. (a) Numerical results; (b) analytic results.

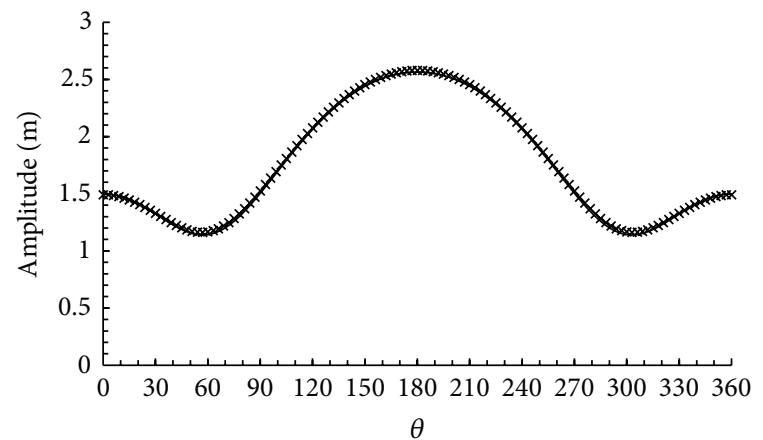

$\times \quad$ Numerical solution

- Analytic solution

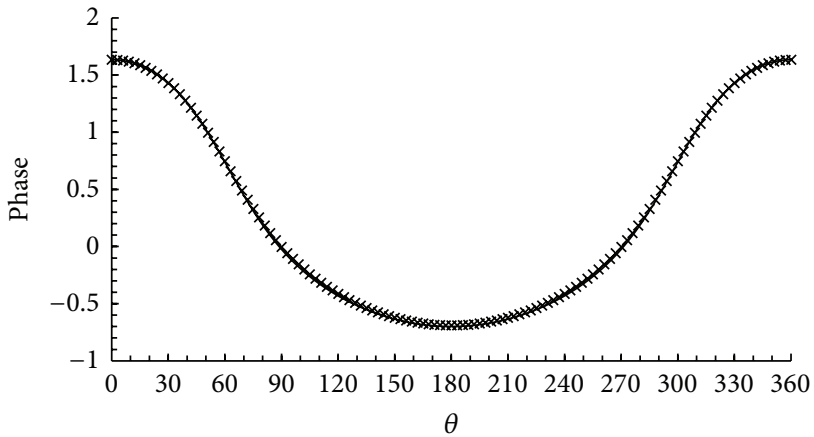

$\times \quad$ Numerical solution

— Analytic solution

(a)

(b)

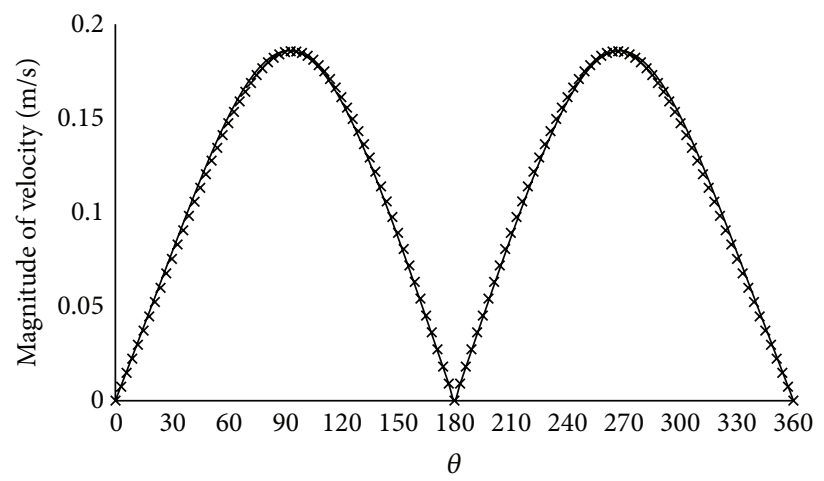

$\times$ Numerical solution

— Analytic solution

(c)

FIgURE 23: Numerical and analytic results of amplitude, phase, and magnitude of velocity around at $r=a$. 


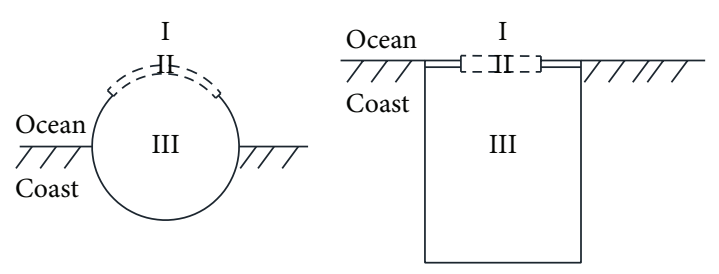

FIGURE 24: Regional connectivity of subdomains for closest nodal searching.

required to provide the connectivity information of the computational nodes and meshes in FEM. Secondly, numerical models with linear shape functions fall short of providing velocity fields of the water-wave motions.

For water-wave combined refraction and diffraction problems with abrupt change of geometry in computational domains, such as existence of breakwater(s), a concept of subdomains of regional connectivity has been established to guarantee application of local collocation that is performed within appropriate subdomains in applications of MFPM. The governing equation and boundary conditions can be referred to as the same of (43)-(45).

Protruding breakwaters or sharp geometrical corners exist in most harbors and may behave like a branch-cut line to divide the water area into subdomains with different characters such as up-wave and down-wave sides, respectively. In the collocation method of MFPM, the approximate polynomial function is established by identifying mostly and closely nearby computational grids in relative distances to a base point. When a protruding breakwater exists, special care must be taken to avoid identifying grid points from different subdomains in this searching process to evaluate coefficients of local polynomials in collocation approximation. Therefore, the computational domain is divided into subdomains and regional connectivity is specified for further local polynomial approximation at each node. In diffraction of water-wave problems, a computational domain can be divided into three subdomains which are the region of ocean, the entrance region of the harbor, and the basin region, shown as in Figure 24 for circular and rectangular harbor, respectively. In searching mostly closed nodes, only nodes in its own and adjacent subdomains indicated by regional connectivity information are included. For example, in Figure 24, when collocation approximation is applied to subdomain I, only nodes in subdomains I and II are considered. For subdomain III, only nodes in subdomains II and III are taken into account to search some closest modes. For subdomain II, all of nodes in its own and adjacent subdomains, namely, II, I, and III, are considered.

A circular harbor with protruding breakwaters into the ocean side is considered, as shown in Figure 25. In this benchmark problem, when monochromatic incident waves propagating into a circular harbor with protruding breakwaters, Mei and Petroni [74] had obtained analytical solutions for wave responses within the region of harbor, as shown in (47)-(49) as follows:

$$
\eta_{H}=a_{0} \frac{N}{D} \sum_{n=0}^{\infty} \frac{\varepsilon_{n}}{2 \pi} \frac{J_{0}(n \delta)}{k a J_{n}^{\prime}(k a)} J_{n}(k r) \cos n\left(\theta-\theta_{I}\right),
$$

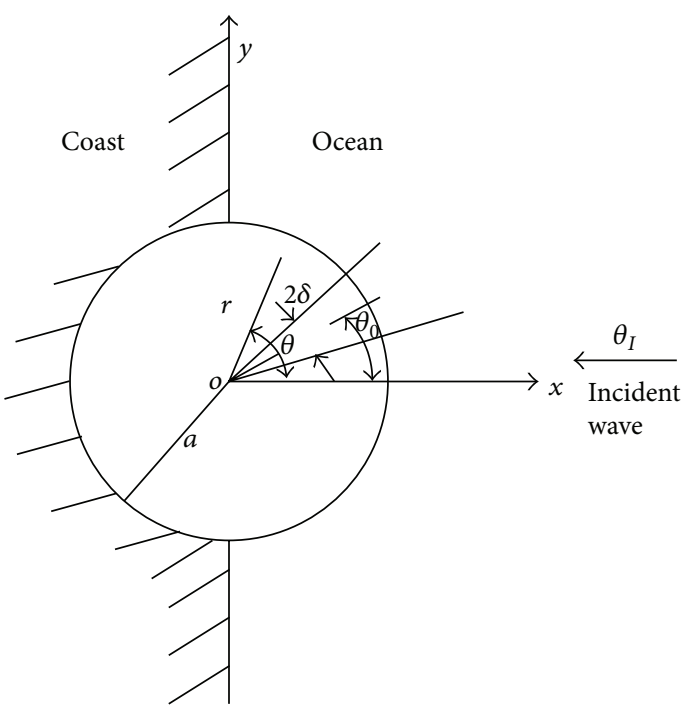

FIGURE 25: Definition of a circular harbor with protruding breakwaters.

where $\eta_{H}$ is water free-surface displacement within the harbor, $a_{0}$ is incident wave amplitude, $\delta$ is half of the harbor entrance opening angle, $\theta_{0}$ is the angle from the positive $x$-coordinate to the centerline of entrance opening, $\theta_{I}$ is direction of incident waves with respect to positive $x$-axis, $a$ is radius of the circular harbor, $k$ is incident wave number, and $N$ is weighted average of the forcing pressure:

$$
\begin{aligned}
N= & \sum_{n=0}^{\infty} \frac{2 i}{\pi k a} \frac{2 \varepsilon_{n} J_{0}(n \delta)}{H_{n}^{\prime}(k a)} \\
& \times\left(\cos \frac{n \pi}{2} \cos n \theta_{I} \cos \theta_{0}+i \sin \frac{n \pi}{2} \sin n \theta_{I} \sin n \theta_{0}\right),
\end{aligned}
$$

and $D$ is term of the coefficient:

$$
\begin{aligned}
D= & \sum_{n=0}^{\infty} \frac{\varepsilon_{n}}{2 \pi} \frac{J_{n}(k a) J_{0}^{2}(n \delta)}{k a J_{n}^{\prime}(k a)} \\
& -\sum_{n=0}^{\infty} \frac{\varepsilon_{n}}{2 \pi} \frac{H_{n}(k a) J_{0}^{2}(n \delta)}{k a H_{n}^{\prime}(k a)}\left(1+\cos n \pi \cos 2 n \theta_{0}\right) .
\end{aligned}
$$

In this problem, verifications of numerical calculations will be presented by comparisons with analytical results of Mei \& Petroni [74]. Two protruding breakwaters into the ocean side clearly separate wave field into two distinct regions. A bridging subdomain at the harbor entrance connects numerical computational information between ocean and harbor sides in present local collocation approach. There are total 6,336 computational nodes used for ocean side (subdomain I), 10 nodes at the harbor mouth (subdomain II), and 449 nodes for the harbor region (subdomain III). The total computational nodes are 6,795. The computational time consumes about 5 minutes of CPU time for one frequency case with computer facility of the personal computer with the Intel(R) Core(TM)2 duo CPU E7300 (2.6 GHz 2.6 GHz) and Microsoft Windows 32 bits system. 


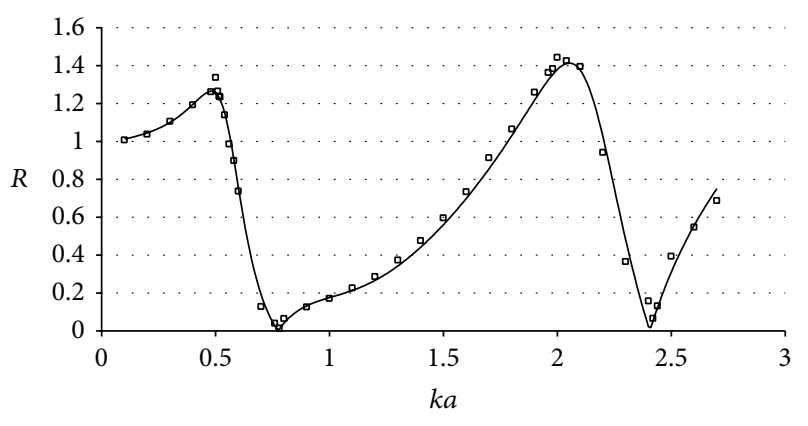

_ Analytical (Mei \& Petroni, 1973)

- Numerical (present)

Figure 26: Response curve at $r=0.7 \mathrm{ft}, \theta=0^{\circ}$ of a circular harbor of radius, $a=0.75 \mathrm{ft}, 60^{\circ}$ opening for $\theta_{I}=225^{\circ}$.

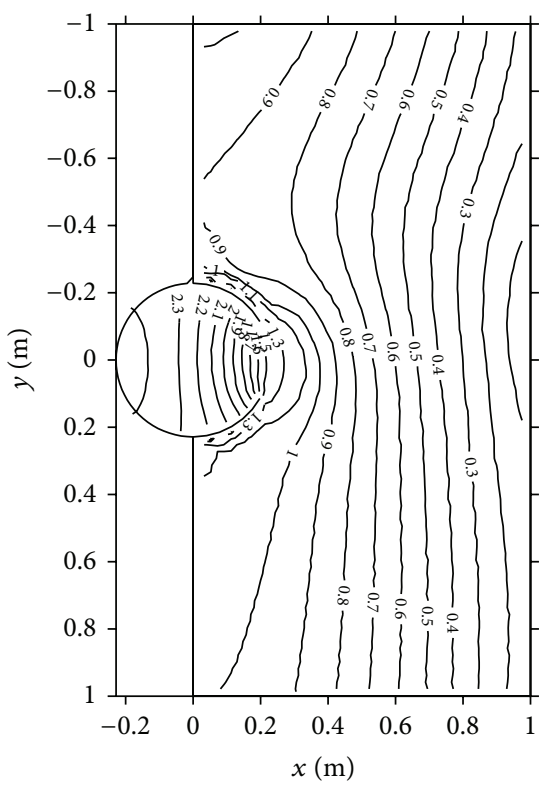

FIgURE 27: Contours of wave amplitude of a circular harbor of radius, $a=0.75 \mathrm{ft}, 60^{\circ}$ opening, with protruding breakwaters at $k a=0.5$ for $\theta_{I}=225^{\circ}$.

A case of this application with incident wave direction $\theta_{I}$ of $225^{\circ}$ is presented. Present numerical results for an idealized circular harbor of radius $a=0.75 \mathrm{ft}$ with harbor mouth $60^{\circ}$ opening $(2 \delta)$.

Response curves at $r=0.70 \mathrm{ft}, \theta=0^{\circ}$, are shown in Figure 26. It is observed that there is very good agreement between present MFPM results and analytical solutions [74]. In the case of these obliquely incident waves, one major resonant wavenumber happens at $k a=0.5$ and the amplification factor, $R$, is equal to 1.46 . At the resonant wavenumber, the corresponding wavelength and period are equal to $2.87 \mathrm{~m}$ and $1.37 \mathrm{~s}$, respectively. Contours of wave amplitude, Figure 27, clearly show different features of wave fields in the up- and down-wave sides, respectively, due to the sheltering effects of the breakwaters. The maximum wave amplitude occurs at the most inner end of the circular harbor.
Velocity distributions of the harbor at $k a=0.5$ indicate the flow directions of the obliquely incident waves at two different time instants, Figure 28. The maximum velocity happens again at the harbor entrance.

4.2.3. Kelvin Waves. For waves with very long periods, such as tidal waves, the Coriolis effects cannot be ignored. Based on the mild-slope equation, an extended mild-slope equation has been formulated to take the effects of Coriolis effects into account, when wave period becomes very long or the domain of concerns is large [73]:

$$
\nabla \cdot(P G \nabla \eta)+\lambda^{2} P G\left(1-\frac{f^{2}}{\omega^{2}}\right) \eta=0,
$$

where $f$ represents the Coriolis parameter and is rotating in a counter-clockwise direction in the northern hemisphere; $\omega$ represents frequency of incident wave; $\lambda$ is the eigenvalue of the characteristic equation, $\omega^{2}=g \lambda \tanh \lambda h$, which may be real or imaginary values; $P$ and $G$ represent, respectively, the wave and group celerity as

$$
\begin{gathered}
P=\frac{\omega}{\lambda} \\
G=\frac{1}{2}\left(1+\frac{2 \lambda h}{\sinh 2 \lambda h}\right) P,
\end{gathered}
$$

and $k$ is the wave number which could be expressed as

$$
k^{2}=\lambda^{2}\left(1-\frac{f^{2}}{\omega^{2}}\right) .
$$

For real value of $\lambda, k$ is a real value when $f<\omega$, there exists a propagating mode of waves, namely, Kelvin waves. For waves with shorter periods, it reduces the traditional mildslope equation $[65,66]$ when Coriolis parameter is negligible.

Pedlosky [75] had studied the case of Kelvin waves by using the linearized momentum equations expressed as

$$
\begin{gathered}
\frac{\partial u}{\partial t}-f v=-g \frac{\partial \varsigma}{\partial x} \\
\frac{\partial v}{\partial t}+f u=-g \frac{\partial \varsigma}{\partial y} \\
\frac{\partial w}{\partial t}=-g \frac{\partial \varsigma}{\partial z} .
\end{gathered}
$$

In this section, Coriolis effects on long waves propagating in an infinite channel are investigated. The channel has two parallel side vertical walls of width $L$, which is shown in Figure 29. Water depth is $H_{0}$ and waves propagating from the left to the right.

According to Pedlosky [75], the analytic solution in Kelvin wave could be expressed as

$$
\begin{gathered}
\eta=\operatorname{Re}\left[\eta_{o} e^{-f y / C_{o}} e^{i \lambda x}\right\} \\
u=\frac{\eta_{0}}{H_{0}} C_{0} e^{-f y / C_{0}} \cos (k x \pm \omega t+\varphi),
\end{gathered}
$$

where $C_{0}=\left(g H_{0}\right)^{1 / 2} ; H_{0}$ is constant depth of the channel; $g$ is the gravity; $\eta_{0}$ is a constant amplitude of incident wave; $\lambda$ is the wave number without effect of Coriolis force. 


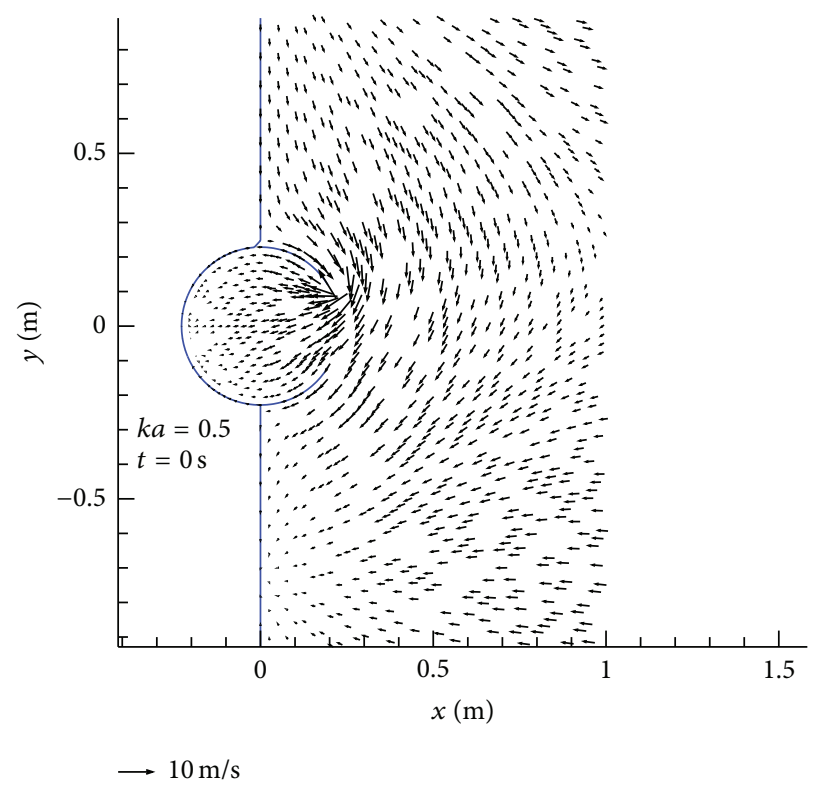

(a)

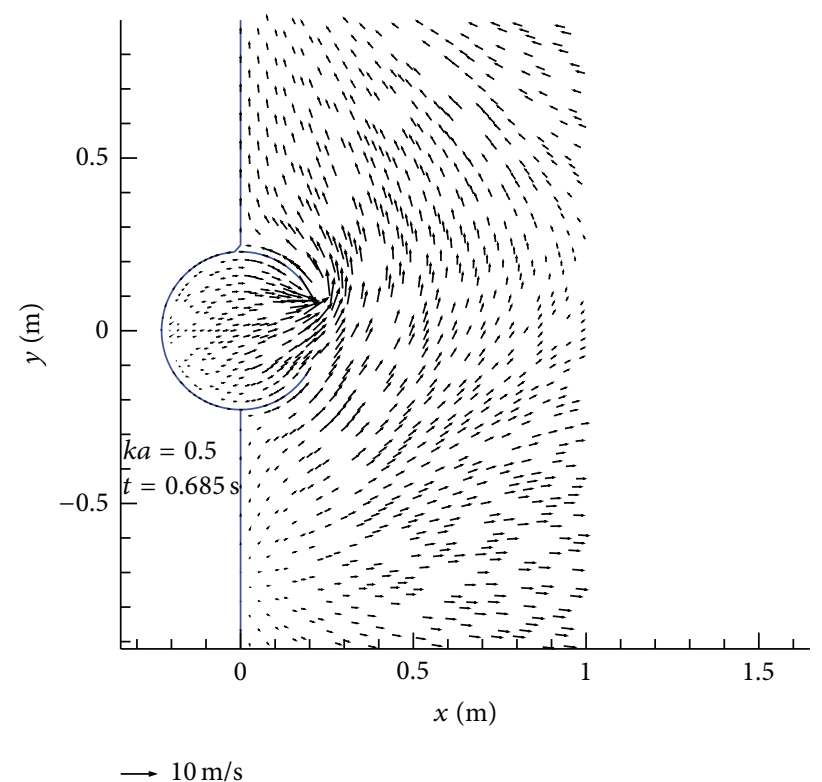

(b)

FIGURE 28: Velocity vectors of a circular harbor of radius, $a=0.75 \mathrm{ft}, 60^{\circ}$ opening, with protruding breakwaters at $k a=0.5$. (a) $t=0$, (b) $t=T / 2$ for $\theta_{I}=225^{\circ}$.

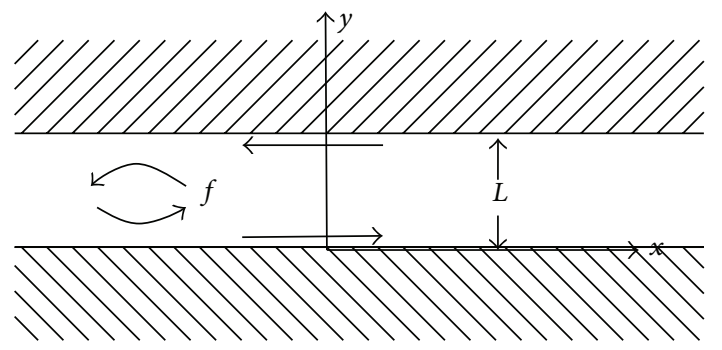

FIGURE 29: An infinite channel of width $L$, with Coriolis parameter $f$.

For numerical computations of MFPM, both radiation condition at the far-field open boundary and no-flux condition on the solid boundaries need to be modified accordingly. Based on the wave modulation of a vertical distribution with $F(z)=\cosh \lambda(z+h) / \cosh \lambda h$, the fluid displacement, $\varsigma$, can be expressed as

$$
\begin{gathered}
\varsigma(x, y, z ; t)=\eta(x, y) F(z) e^{-i \omega t} \\
\eta(x, y)=A(x, y) e^{i \int k_{n} \cdot d \vec{x}}=A(x, y) e^{i \int\left(k_{x} d x+k_{y} d y\right)},
\end{gathered}
$$

where $A$ is amplitude of free surface displacement, $\eta ; k$ is wave number; $k_{x}$ and $k_{y}$ are the components of $k$ in $x$ - and $y$ direction, respectively.

Assuming that the flow velocities associated with wave action, $(u, v)$, also have the same vertical distribution and with $(U, V)$ as the components at the free surface:

$$
(u, v)=(U, V) F(z) .
$$

From (53) and (55)-(56), the velocity components formula with Coriolis effects can be derived and expressed as

$$
\begin{gathered}
U=\frac{g f}{\omega^{2}-f^{2}} \frac{\partial \eta}{\partial y}-\frac{i g \omega}{\omega^{2}-f^{2}} \frac{\partial \eta}{\partial x} \\
V=-\frac{g f}{\omega^{2}-f^{2}} \frac{\partial \eta}{\partial x}-\frac{i g \omega}{\omega^{2}-f^{2}} \frac{\partial \eta}{\partial y} .
\end{gathered}
$$

Then from the no-flux condition, $\vec{U} \cdot \vec{n}=0$, the solid boundary condition could be expressed as

$$
\frac{\partial \eta}{\partial n}= \pm \frac{i f}{\omega} \frac{\partial \eta}{\partial s},
$$

where $n$ and $s$ represent normal and tangent direction on the solid boundary, respectively. It is noted that the modified no-flux condition, (59), reduces to a traditional one when Coriolis parameter is equal to zero.

For practical purposes, outgoing propagation of scattered waves satisfy the Sommerfeld [55] radiation condition as (31) can be approximated as a local one as

$$
\frac{\partial \eta^{S}}{\partial n}-i \lambda \eta^{s}=0
$$

In order to illustrate the Coriolis effects of waves propagating in a channel with width $L=60 \mathrm{~km}$ and water depth $H_{0}=3 \mathrm{~m}$ have been used. A wave period $T=$ $46800 \mathrm{sec}$, amplitude $=1.0 \mathrm{~m}$, and Coriolis parameter $f=$ $0.0000935638 \mathrm{sec}^{-1}$ (equivalently at latitude $=40^{\circ}$ ) have been taken. The wave in the channel is propagating from the left to right. The result of analytical solution is shown as in Figure 30. The distribution of wave amplitude decreases exponentially 


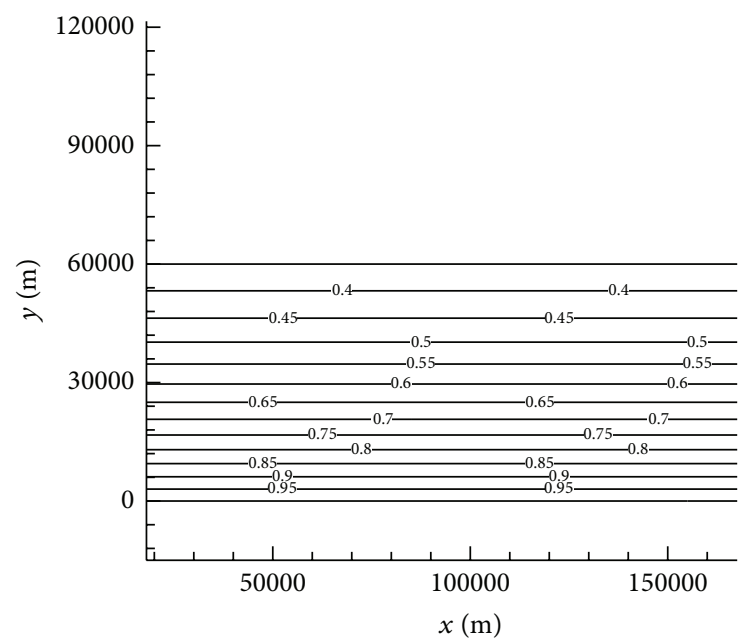

(a)

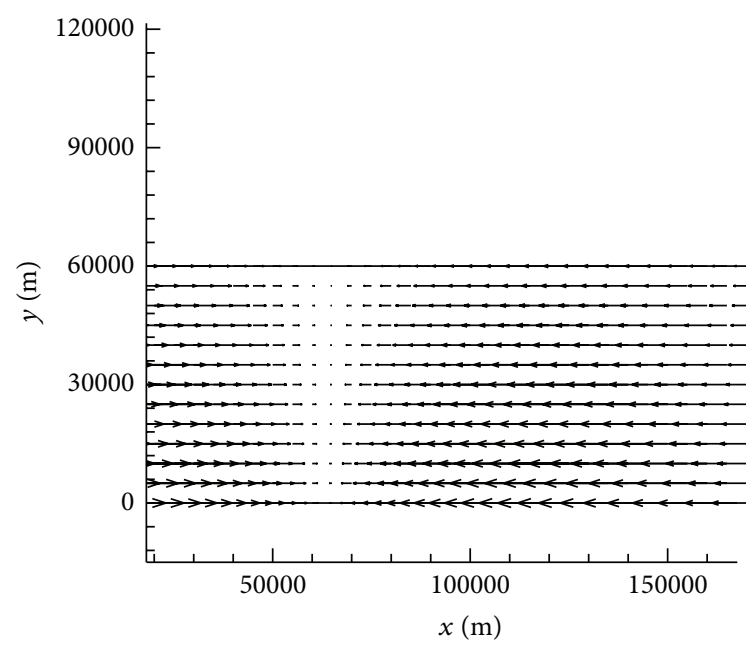

(b)

FIGURE 30: The results of analytical solutions: (a) distribution of wave amplitude; (b) distribution of wave velocity.

from right to left for an observer looking in the same direction of wave propagation. The rate of decrease of the amplitude from right to left is proportional to $c / f$, the Rossby radius of deformation. The distribution of the wave velocity is parallel to the walls of the channel and also proportional to $c / f$.

For numerical computations, the incident wave amplitude is chosen to be $\eta_{o} e^{-f y / C_{o}}$ and propagates from left to right. The length of the channel of $1,000 \mathrm{~km}$ was used for numerical computations. The local radiation boundary condition as (59) was used on the other end of the channel for the incident wave and scatter wave. By applying this numerical MFPM model, the velocity at each node can be also obtained simultaneously with the free surface displacement. Present numerical results of amplitude and velocity, Figure 31, show very good agreement with analytical solutions. The amplitude and velocity of $x$-direction between the numerical and analytical results at cross section $x=10,000 \mathrm{~m}$ are further compared as shown in Figure 32. Very good agreement between numerical and analytical results is observed. The velocity of $y$-direction is equal to 0 with a negligible error. Very good agreement between numerical results and analytical solutions is observed.

4.3. Time-Domain Water Wave Problems. For several decades, free-surface water wave problems have been studied as potential-flow problems governed by the Laplace equation with nonlinear free surface boundary conditions. If the motions of the free-surface are periodic, the problems can be treated as frequency-domain problems as illustrated above. But if one wants to obtain more detail in the transient process of the wave motion, time-domain water wave problems are recommended to be considered.

Most of these studies have been carried out with boundary-element methods (BEM), subjected to a mixed Eulerian-Lagrangian (MEL) time marching approach [7681]. The governing equation is the Laplace equation

$$
\nabla^{2} \phi=0
$$

where $\phi(\vec{x}, t)$ is the velocity potential with $\vec{x}$ being the position vector and $t$ being the time. The velocity vector is defined as $\vec{v}=\nabla \phi((\partial \phi / \partial x) \vec{i}+(\partial \phi / \partial y) \vec{j}$ for 2-D problems and $(\partial \phi / \partial x) \vec{i}+(\partial \phi / \partial y) \vec{j}+(\partial \phi / \partial z) \vec{k}$ for 3-D problems). The kinematic free-surface boundary condition (KFSBC) and dynamic free-surface boundary condition (DFSBC) can be expressed as

$$
\begin{gathered}
\nabla \phi=\frac{d \vec{x}}{d t}, \quad \vec{x} \in \text { the entire domain, } \\
\text { including the free surface, } \\
\frac{d \phi}{d t}=-g z+\frac{1}{2} \nabla \phi \cdot \nabla \phi, \quad \vec{x} \in \text { the free surface, }
\end{gathered}
$$

where $g$ is the gravitational acceleration, $z$ is the vertical coordinate, and $C(t)$ is Bernoulli's constant, which can be set to zero for a quiescent ambiance. Both the KFSBC and the DFSBC are stated in the Lagrangian frame. The boundary condition at the water-body interface is the no-flux boundary condition, which can be expressed as

$$
\vec{n} \cdot \nabla \phi=\vec{n} \cdot \vec{v}_{b},
$$

where $\vec{n}$ is the unit normal vector outward from the body surface, and $\vec{v}_{b}$ is the velocity of the body surface. The righthand side of this equation equals zero for a fixed bottom in a finite depth and will be given by the motion of the solid boundary.

For solving this time dependent problem, the timedomain firstly has to be discretized. At each time step, the Laplace equation needs to be solved once to obtain the velocity potential for the entire domain thus to get the velocity. Boundary conditions are updated by the motion of the solid boundary and prediction from the free-surface boundary, which involves nonlinear terms. One could use the a mixed Eulerian-Lagrangian (MEL) time marching 


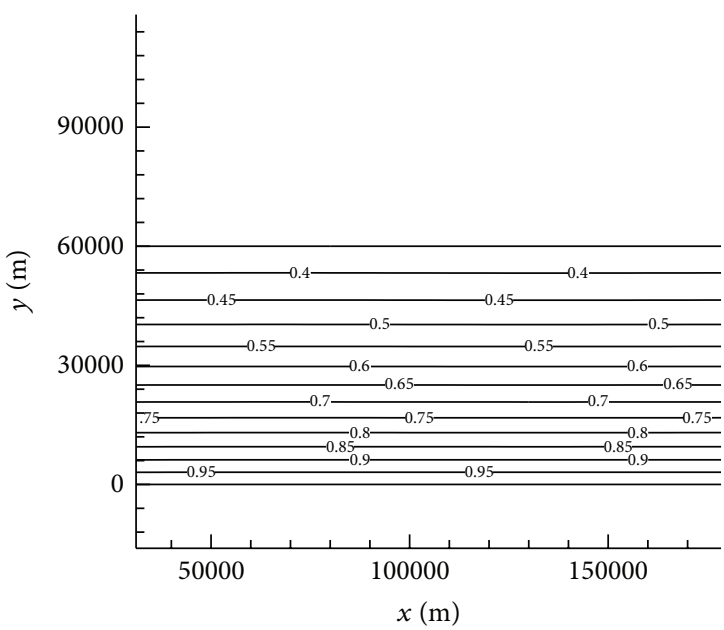

(a)

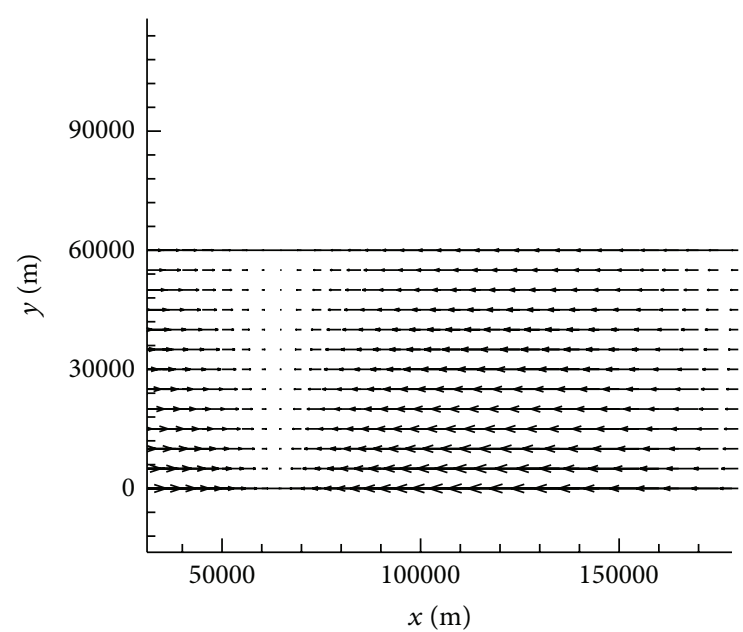

(b)

FIGURE 31: The results of numerical model: (a) distribution of wave amplitude; (b) distribution of wave velocity.

approach in which the 4th order Runge-Kutta time integration is used, or choose a simpler but satisfactory time marching scheme proposed in [21]. Adopting the time marching scheme proposed in [21], the DFSBC (66) can be formulated as

$$
\begin{aligned}
\left(\left.\phi\right|_{\vec{x}=\vec{x}_{j}}\right)^{(n)}= & \left(\left.\phi\right|_{\vec{x}=\vec{x}_{j}}\right)^{(n-2)} \\
& +2 \Delta t\left[\left(-g z+\frac{1}{2} \nabla \phi \cdot \nabla \phi\right)_{\vec{x}=\vec{x}_{j}}\right]^{(n-1)},
\end{aligned}
$$

where $\vec{x}_{j}$ denotes the position of the $j$ th node and this equation is only valid in case the node is on the free surface. In this formulation, the required data on the right-hand side for seeking the velocity potential in the entire domain at the $n$th time step, including the nonlinear terms, are already known. Generally speaking, this explicit time marching scheme may be regarded as a leap-frog approach. What one needs to do first is just to determine the position of each traced "particle," $\vec{x}_{j}^{(n)}$. It was proposed to use the second-order finite difference scheme in the time-domain, also a leap-frog approach, to determine the position of $\vec{x}_{j}^{(n)}$ :

$$
\vec{x}_{j}^{(n)}=\vec{x}_{j}^{(n-2)}+2 \Delta t\left(\left.\nabla \phi\right|_{\vec{x}_{=} \vec{x}_{j}}\right)^{(n-1)} \text {. }
$$

In the case of strong nonlinearity, the free-surface particle trajectory $\vec{x}_{j}^{(n)}$ should be modified by applying the CrankNicolson formula for a better numerical stability:

$$
\vec{x}_{j}^{(n)}=\vec{x}_{j}^{(n-1)}+\frac{\Delta t}{2}\left[\left(\left.\nabla \phi\right|_{\vec{x}=\vec{x}_{j}}\right)^{(n)}+\left(\left.\nabla \phi\right|_{\vec{x}=\vec{x}_{j}}\right)^{(n-1)}\right] .
$$

The above equation is applicable after the velocity potential for the entire domain is solved. Note that there is no need to solve the Laplace equation again because there is barely difference between the free-surface velocity potential at $\vec{x}_{j}^{(n)}$ predicted by using (64) and that predicted by using (65). The accuracy of this time marching scheme has been verified in [21, 50-53].

4.3.1. Solitary Wave Generation by a Piston-Type Wave Maker. The first application of the MFPM to transient water wave problem is the simulation of the solitary wave generation by a piston-type wave maker [52]. In [52] a modification of Goring's solitary wave generation method [82] was proposed. The motion of the solid boundary in the model (i.e., (66)) is described as

$$
\frac{d \xi}{d t}=\bar{u}(\xi, t)=\frac{\left.C \eta\right|_{x=\xi}}{h+\left.\eta\right|_{x=\xi}}
$$

where $\xi(t)$ is the position of the wave paddle at time $t$, while $t$ is elapsed time since the start of the motion, $C$ is the wave celerity, $\eta$ is the free surface displacement, and $h$ is the still water depth. In [82] it was proposed to apply Boussinesq's solitary wave solution [83] into (66). That is,

$$
\eta=H S^{2}
$$

where $H$ is the wave height, and

$$
S=\cosh ^{-1}[K X]
$$

in which $K$ is the boundary outskirt decay coefficient, and

$$
X=\left(\xi-C t-x_{0}\right),
$$

where $x_{0}$ is the initial position of the wave crest. Because the solitary wave is generated in a quiescent water flume, $x_{0}$ must be chosen as a negative value, which means the wave crest is initially out of the domain. Its value is chosen by 


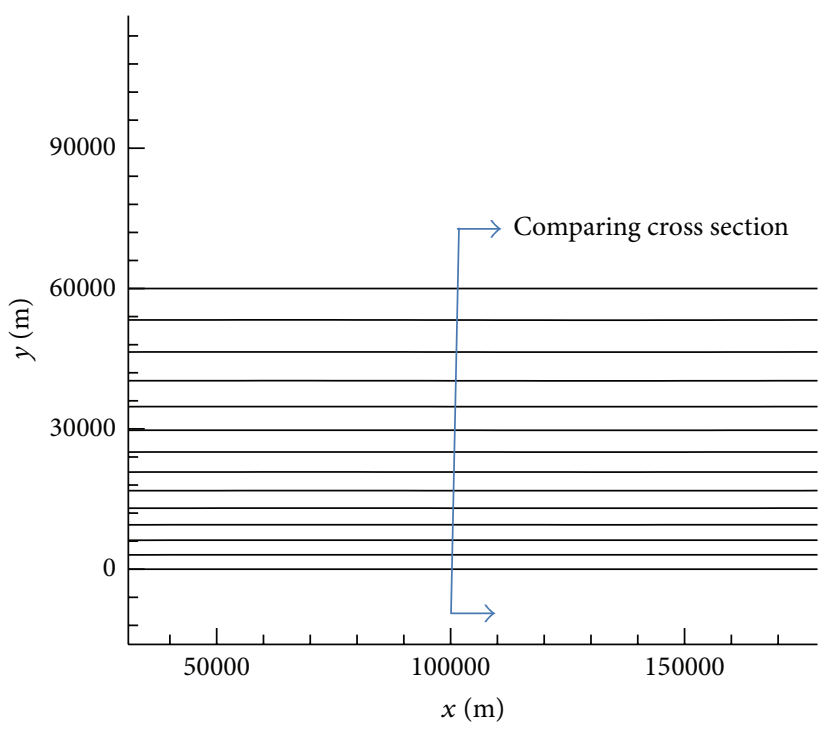

(a)

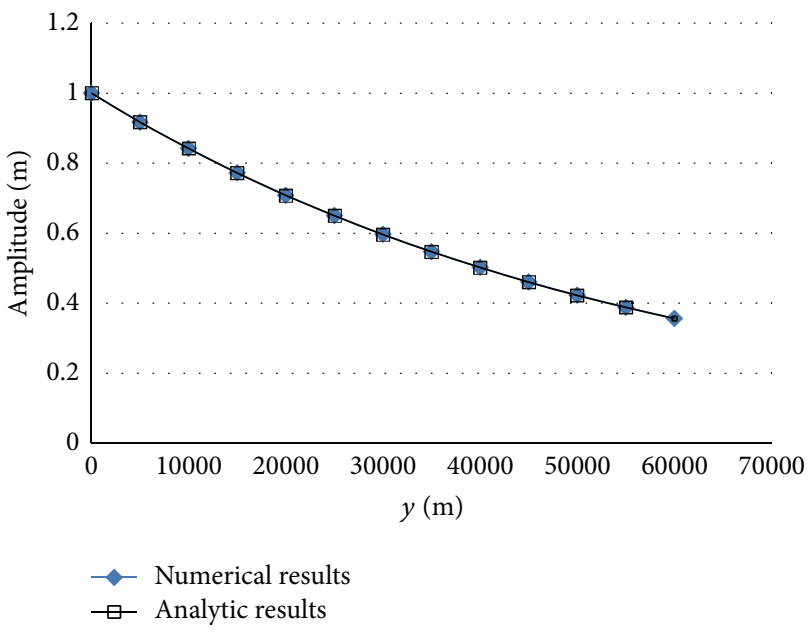

(b)

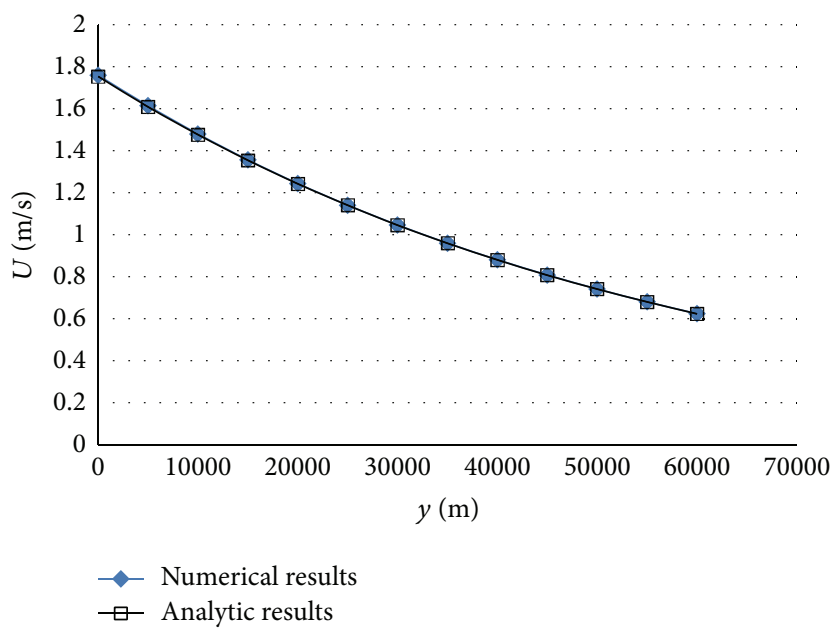

(c)

FIGURE 32: Comparisons of amplitude and velocity at $x$-diretion between numerical and analytical results at the cross section $x=10,000 \mathrm{~m}$.

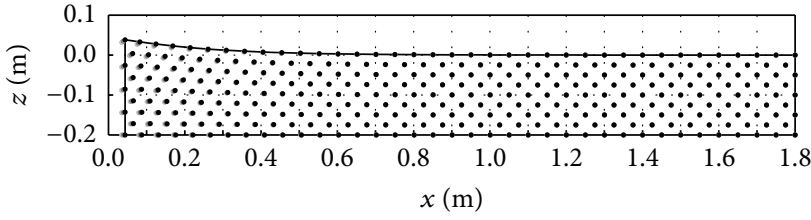

(a)

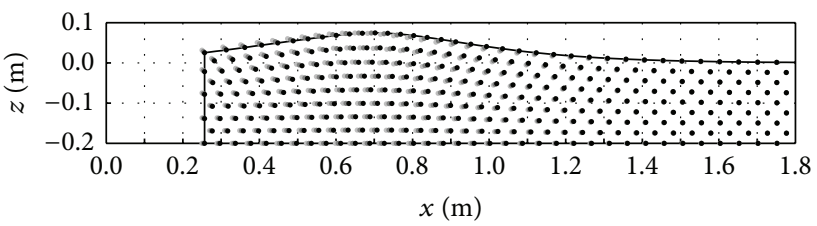

(c)

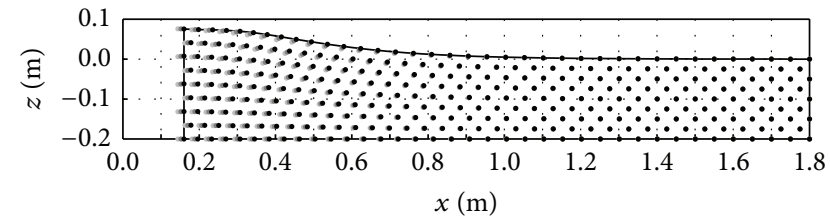

(b)

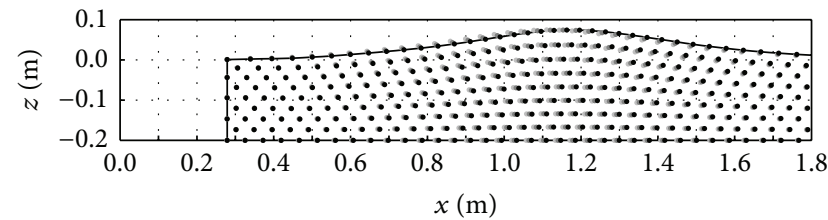

(d)

FIGURE 33: Snapshots of fluid particles during wave generating process, for the case of water depth with $20 \mathrm{~cm}$ and input wave height of $7.4 \mathrm{~cm}$. 


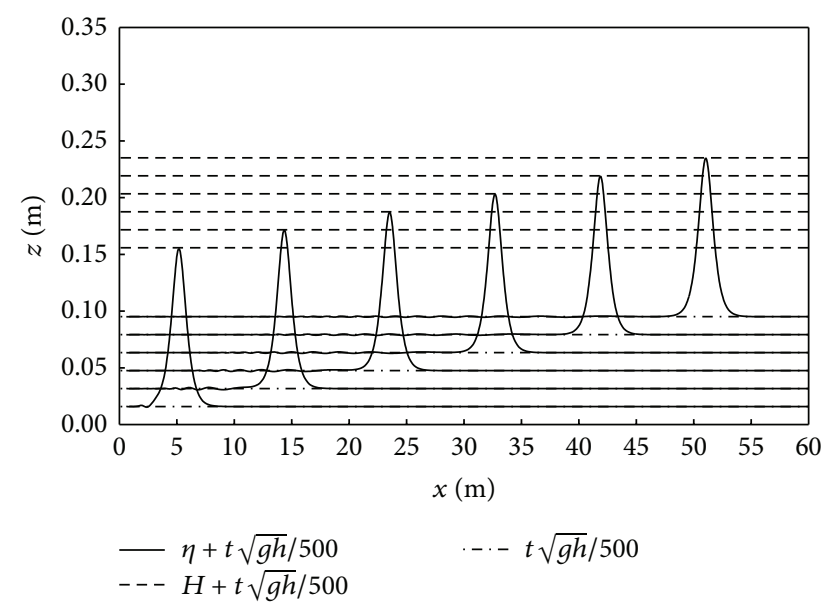

FIGURE 34: Evolution of the free surface wave for the case of $H_{0}=$ $0.14 \mathrm{~m}$ and $h=0.4 \mathrm{~m}$ generated by the new solitary wave generation method. The subscript " 0 " denotes the original wave height setup in the wave generation control.

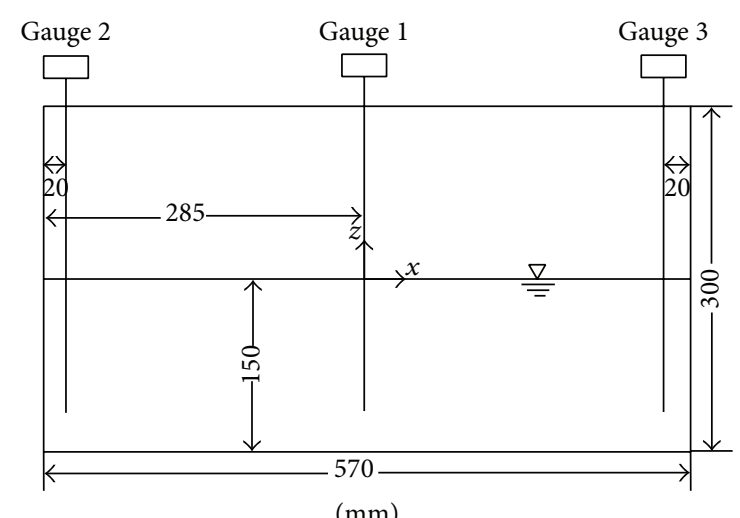

FIgURE 35: Layout of the sloshing experiment of Liu and Lin (2008).

considering the length of the wave. The boundary outskirt decay coefficient of Boussinesq's solitary wave solution is determined as

$$
K=\sqrt{\frac{3 H}{4 h^{3}}},
$$

where the wave celerity is determined as

$$
C=\sqrt{g(h+H)} .
$$

Figure 33 shows the snapshots of fluid particles during the wave generating process. In the case, the water depth is $20 \mathrm{~cm}$ and Goring's solitary wave generation method is used. The input in (71) is $7.4 \mathrm{~cm}$. Details about the setup of this case and the verification of the model could be found in [52]. How the height of the wave crest decays during the wave propagation could also be found in [52].

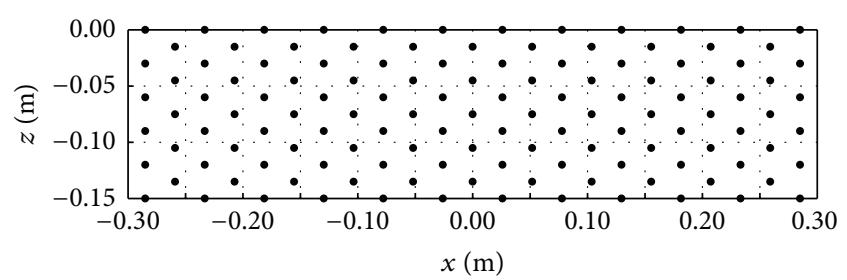

FIGURE 36: Initial nodal distribution of the numerical model of the liquid sloshing in a swaying tank.

In [52] it was suggested that one should apply the 9th order solitary wave solution [84] into (70) so the generated solitary wave could propagate for a longer distance without the wave crest decay. In [84], the free surface displacement can be described as

$$
\eta=h\left(\left(\frac{H}{h}\right) \eta_{1}+\left(\frac{H}{h}\right)^{2} \eta_{2}+\cdots+\left(\frac{H}{h}\right)^{9} \eta_{9}\right) .
$$

The formulae to determine the values of $\eta_{1}$ to $\eta_{9}$ could be found in [84]. In an analogous way, the boundary outskirt decay coefficient and the wave celerity are determined as

$$
\begin{aligned}
& K=\sqrt{\frac{3 H}{4 h^{3}}}\left(1+\left(\frac{H}{h}\right) K_{1}+\left(\frac{H}{h}\right)^{2} K_{2}+\cdots\left(\frac{H}{h}\right)^{9} K_{9}\right), \\
& C^{2}=g h\left(1+\left(\frac{H}{h}\right) C_{1}+\left(\frac{H}{h}\right)^{2} C_{2}+\cdots+\left(\frac{H}{h}\right)^{9} C_{9}\right),
\end{aligned}
$$

where the formulae to determine $K_{1}, \ldots, K_{9}$ and $C_{1}, \ldots, C_{9}$ are all listed in [84].

The numerical results show that by applying the 9 th order solitary wave solution [84] into (70) the wave generated can propagate without decay for a distance hundred times longer than the water depth, as shown in Figure 34.

4.3.2. Liquid Sloshing in a Swaying Tank. A numerical model for simulating the free surface waves of the liquid sloshing in a swaying tank was developed in [53]. The model was verified by the comparison with the experimental data in [85]. The layout of the experiment is shown in Figure 35. This experiment was carried out to verify the numerical model developed in [85] which is a VOF (volume of fluid) model for the simulation of real fluid motion governed by the Reynolds-averaged Navier-Stokes equations. Among several cases carried out in [85], the nonbreaking case with the strongest nonlinear effect in the experiment is chosen as the verification of present model. The period of the oscillation is $1.0372 \mathrm{sec}$. The amplitude of the oscillation is $0.5 \mathrm{~cm}$.

Discretizing a wave length with at least 20 segments, the initial nodal spacing on the free surface is chosen as $5.18 \mathrm{~cm}$. The collocation points are initially distributed as a hexagonal close packing array so that the most compact nodal arrangement can be achieved. Therefore, the vertical nodal spacing on the side walls is $3 \mathrm{~cm}$. Totally, there are 127 collocation points. The time step chosen in the simulation is $1 / 80$ of the swaying period. The initial nodal distribution is shown in Figure 36. 


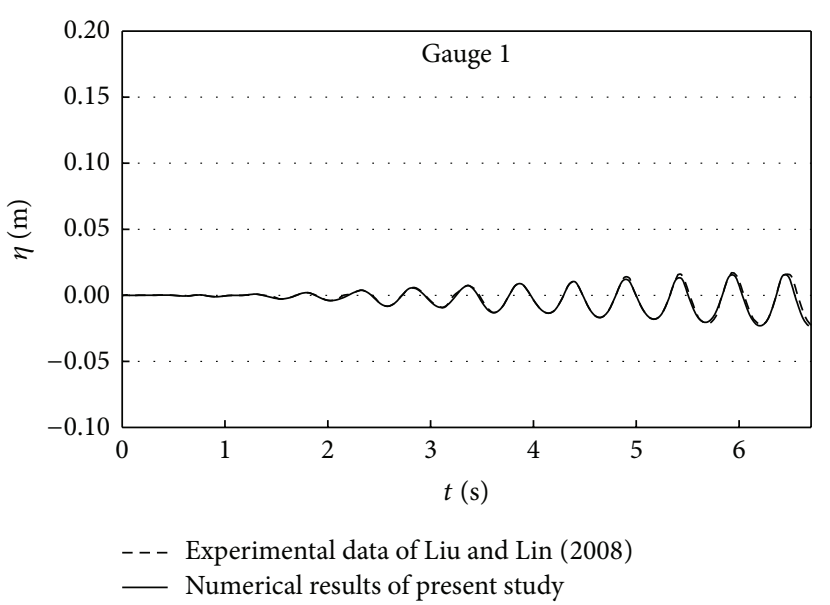

(a)

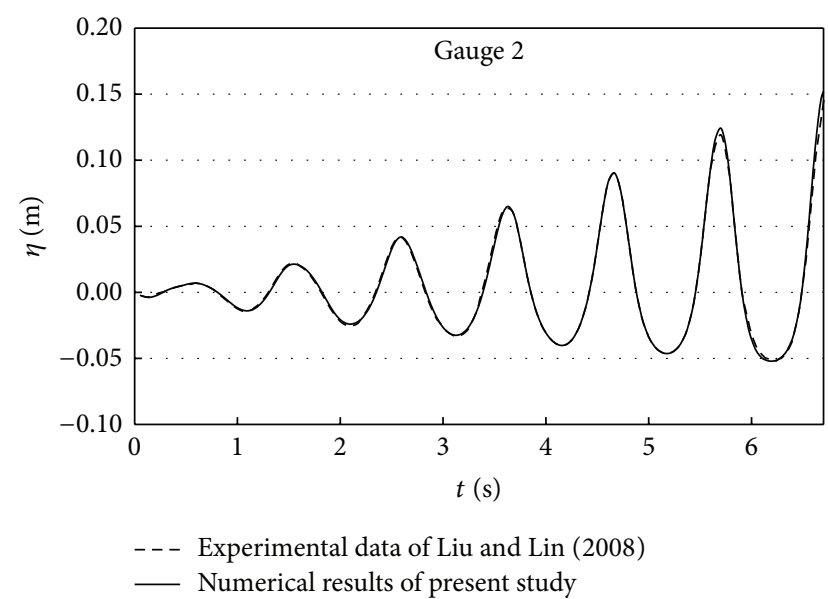

(b)

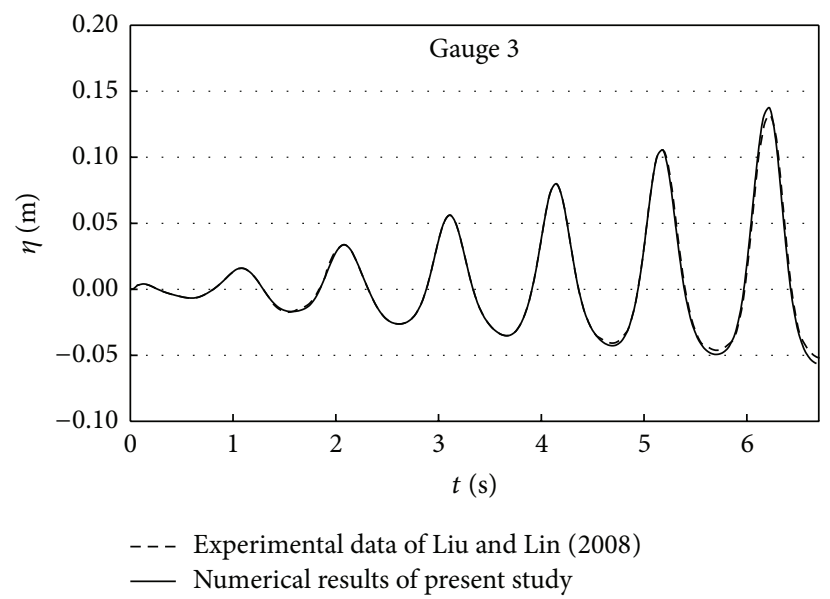

(c)

FIgURE 37: Comparison of the numerical results with the experimental data.

Figure 37 shows the comparison of the numerical results with the experimental data. Very good agreement is found. It is also found that the higher the peak grows, the flatter the trough becomes. This indicates an increase of nonlinear effect as the tank oscillation continues. In the simulation the side walls are set to be infinitely high but in the experiment the tops of the two side walls are just $15 \mathrm{~cm}$ high from the still water level. What happened when the free surface elevation goes higher than the height of side walls had not been mentioned in [85]. In present simulation, free surface elevation goes over the top of the right side wall at $t=$ $6.184 \mathrm{sec}$. This might be the reason why the simulated free surface displacement, $\eta$, in the last wave period is slightly higher than observed. Figure 38 shows the positions of the traced fluid particles in the time interval of $t=6.0 T \sim 6.5 T$. This figure shows that, at the end of simulation, the run-up becomes much higher than the initial water depth. It is an indication of very strong nonlinearity on the free surface. Only 9 seconds is needed to simulate this case by using just one processing unit of Intel(R) Core(TM) i7-3370 CPU. The nodal spacing of present model is an order larger than the grid size in the model of [75]. Present model is much more efficient in this case:

$$
t=6.0 T \sim 6.5 T .
$$

\section{Conclusions}

Recent advancement on the modified finite point method is reviewed in this paper. A normalized parameter in the Gaussian distribution of the weighting factor is presented with recommendation of relevant parameters for better numerical results, regardless of the size of the computational domain. A concept of regional connectivity is introduced and demonstrated for computational domains consisting of different physical behaviors in subdomains. This concept will be needed for similar problems such as a crack in a thin plate and a cutoff wall in groundwater seepage.

Benchmark examples of employing this method to solve Laplace, Poisson, convection-diffusion, Helmholtz, mildslope, and extended mild-slope equations are verified. This 


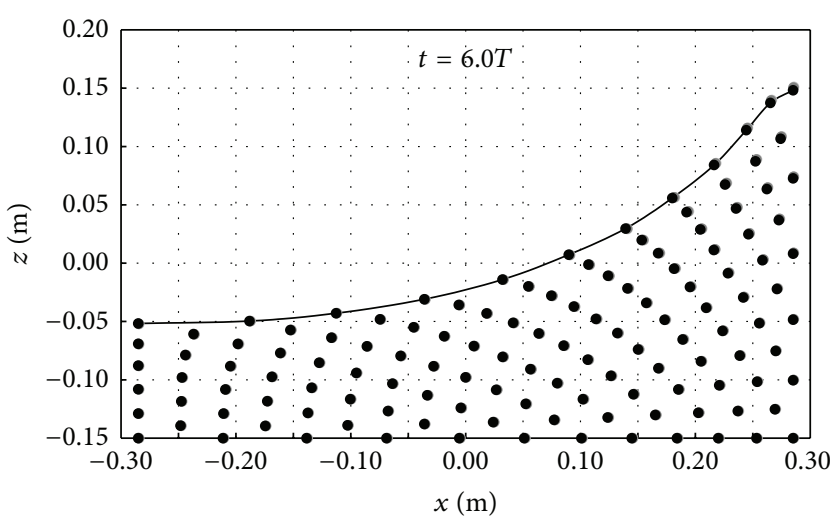

(a)

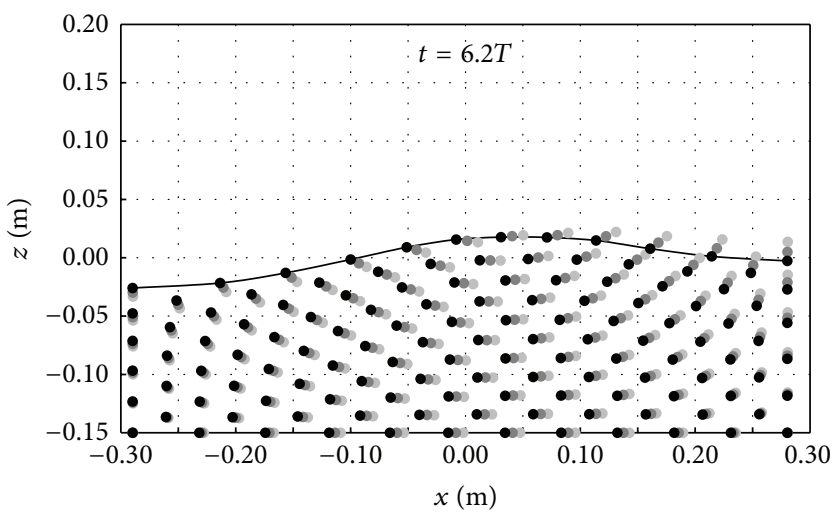

(c)

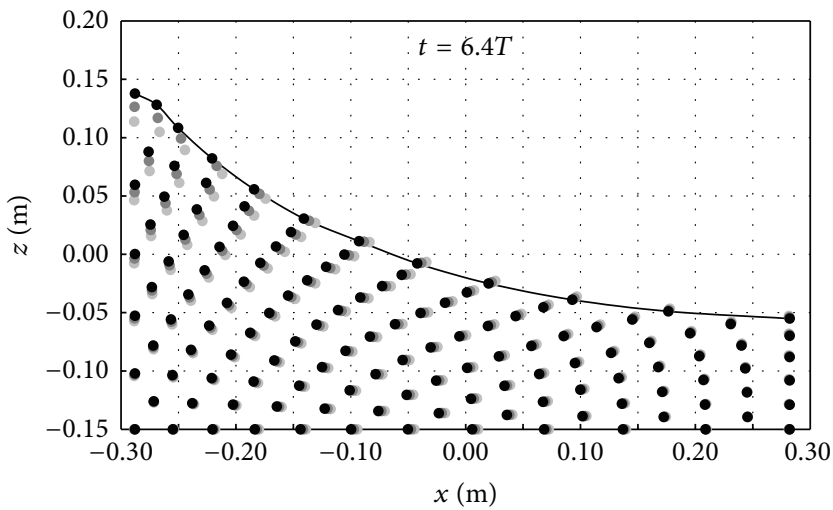

(e)

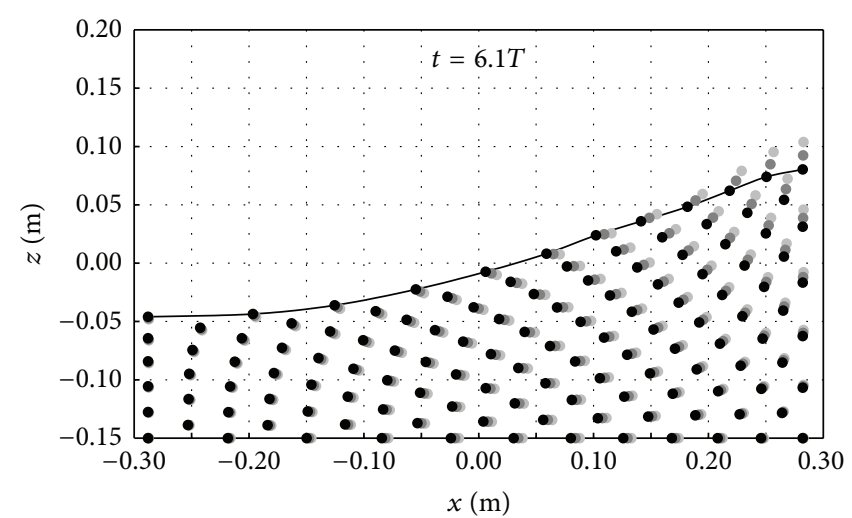

(b)

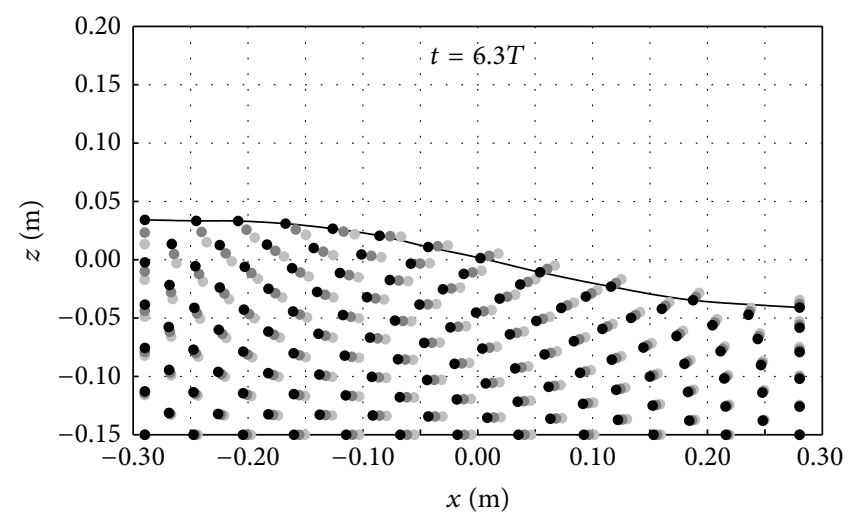

(d)

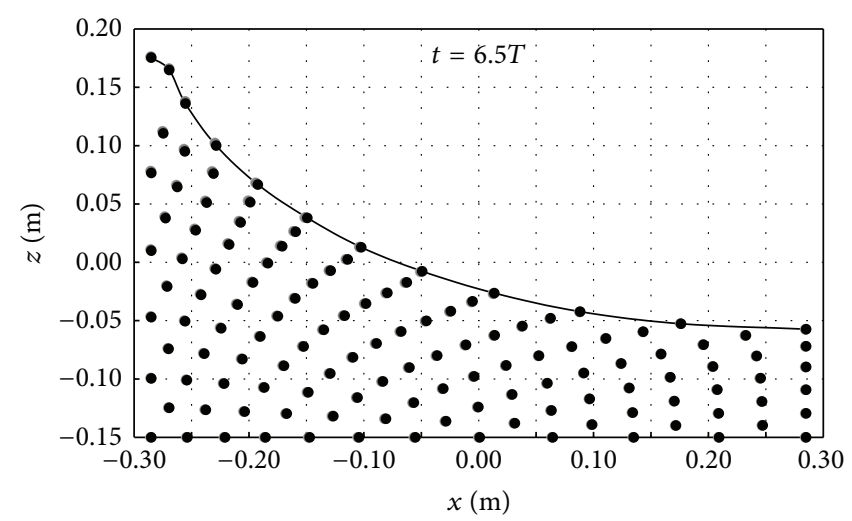

(f)

FIGURE 38: Snapshots of traced fluid particles in the time interval of $t=6.0 T \sim 6.5 T$.

method could be employed to solve governing equations with real-valued unknowns as well as complex ones. Application of MFPM to numerical generation of orthogonal grids is illustrated to overcome difficulty of other methods. Problems of free-surface water waves represented in frequency- and timedomain are demonstrated for the applicability of MFPM. The derivatives of the potentials can be easily obtained as part of the solutions after the local collocations are achieved. This advantage of MFPM will provide great potentials in numerical simulations for physical problems when accurate derivatives of the solution functions are of particular interest.

\section{Conflict of Interests}

The authors declare that there is no conflict of interests regarding the publication of this paper.

\section{Acknowledgment}

The authors would like to thank the National Science Council of Taiwan for the financial support through Projects nos. 1022221-E-002-155 and NSC 103-2911-I-006-302 in these years for developing this MFPM method. 


\section{References}

[1] R. A. Gingold and J. J. Maraghan, "Smoothed particle hydrodynamics: theory and applications to non-spherical stars," Monthly Notices of the Royal Astronomical Society, vol. 181, pp. 375-389, 1977.

[2] G. R. Liu and Y. T. Gu, An Introduction to Meshfree Methods and Their Programming, Springer, 2005.

[3] J. E. Kansa, "Multiquadrics—a scattered data approximation scheme with applications to computational fluid dynamics-II, solutions to parabolic, hyperbolic and elliptic partial differential equations," Computers and Mathematics with Applications, vol. 19, pp. 127-145, 1990.

[4] Y. C. Hon, M. W. Lu, W. M. Xue, and Y. M. Zhu, "Multiquadric method for the numerical solution of a biphasic mixture model," Applied Mathematics and Computation, vol. 88, no. 2-3, pp. 153$175,1997$.

[5] Y. C. Hon, M. W. Lu, W. M. Xue, and X. Zhou, "New formulation and computation of the triphasic model for mechanoelectrochemical mixtures," Computational Mechanics, vol. 24, no. 3, pp. 155-165, 1999.

[6] M. Zerroukat, H. Power, and C. S. Chen, "A numerical method for heat transfer problem using collocation and radial basis functions," International Journal for Numerical Methods in Engineering, vol. 42, no. 7, pp. 1263-1278, 1998.

[7] Y. C. Hon and X. Z. Mao, "An efficient numerical scheme for Burgers' equation," Applied Mathematics and Computation, vol. 95, no. 1, pp. 37-50, 1998.

[8] Y. C. Hon, K. F. Cheung, X. Z. Mao, and E. J. Kansa, "Multiquadric solution for shallow water equations," Journal of Hydraulic Engineering, vol. 125, no. 5, pp. 524-533, 1999.

[9] S. M. Wong, Y. C. Hon, and M. A. Golberg, "Compactly supported radial basis functions for shallow water equations," Applied Mathematics and Computation, vol. 127, no. 1, pp. 79101, 2002.

[10] X. Zhou, Y. C. Hon, and K. F. Cheung, "A grid-free, nonlinear shallow-water model with moving boundary," Engineering Analysis with Boundary Elements, vol. 28, no. 8, pp. 967-973, 2004.

[11] D. L. Young, S. C. Jane, C. Y. Lin, C. L. Chiu, and K. C. Chen, "Solutions of $2 \mathrm{D}$ and $3 \mathrm{D}$ stokes laws using multiquadrics method," Engineering Analysis with Boundary Elements, vol. 28, no. 10, pp. 1233-1243, 2004.

[12] D. L. Young, C. S. Chen, and T. K. Wong, "Solution of Maxwell's equations using the MQ method," Computers, Materials and Continua, vol. 2, no. 4, pp. 267-276, 2005.

[13] S. J. Lai, B. Z. Wang, and Y. Duan, "Meshless radial basis function method for transient electromagnetic computations," IEEE Transactions on Magnetics, vol. 44, no. 10, pp. 2288-2295, 2008.

[14] Y. C. Hon and X. Z. Mao, "A radial basis function method for solving options pricing model," Financial Engineering, vol. 8, pp. 31-49, 1999.

[15] M. D. Marcozzi, S. Choi, and C. S. Chen, "On the use of boundary conditions for variational formulations arising in financial mathematics," Applied Mathematics and Computation, vol. 124, no. 2, pp. 197-214, 2001.

[16] B. Fornberg, T. A. Driscoll, G. Wright, and R. Charles, "Observations on the behavior of radial basis function approximations near boundaries," Computers \& Mathematics with Applications, vol. 43, no. 3-5, pp. 473-490, 2002.
[17] N. Mai-Duy and T. Tran-Cong, "Approximation of function and its derivatives using radial basis function networks," Applied Mathematical Modelling, vol. 27, no. 3, pp. 197-220, 2003.

[18] N. J. Wu, T. K. Tsay, and D. L. Young, "Meshless simulation for fully nonlinear water waves," International Journal for Numerical Methods in Fluids, vol. 50, pp. 219-234, 2006.

[19] N. Wu, T. Tsay, and D. L. Young, "Computation of nonlinear free-surface flows by a meshless numerical method," Journal of Waterway, Port, Coastal and Ocean Engineering, vol. 134, no. 2, pp. 97-103, 2008.

[20] N. J. Wu and T. K. Tsay, "Applicability of the method of fundamental solutions to 3-D wave-body interaction with fully nonlinear free surface," Journal of Engineering Mathematics, vol. 63, no. 1, pp. 61-78, 2009.

[21] N. Wu and K. Chang, "Simulation of free-surface waves in liquid sloshing using a domain-type meshless method," International Journal for Numerical Methods in Fluids, vol. 67, no. 3, pp. 269288, 2011.

[22] N. Perrone and R. Kao, "A general finite difference method for arbitrary meshes," Computers and Structures, vol. 5, no. 1, pp. 45-57, 1975.

[23] E. Oñate, S. Idelsohn, O. C. Zienkiewicz, and R. L. Taylor, "A finite point method in computational mechanics. Applications to convective transport and fluid flow," International Journal for Numerical Methods in Engineering, vol. 39, no. 22, pp. 38393866, 1996.

[24] E. Oñate, S. Idelsohn, O. C. A. Zienkiewicz, and C. Sacco, "A stabilized finite point method for analysis of fluid mechanics problems," Computer Methods in Applied Mechanics and Engineering, vol. 139, no. 1-4, pp. 315-346, 1996.

[25] C. K. Lee, X. Liu, and S. C. Fan, "Local multiquadric approximation for solving boundary value problems," Computational Mechanics, vol. 30, no. 5-6, pp. 396-409, 2003.

[26] T. J. Liszka, C. A. M. Duarte, and W. W. Tworzydlo, " $h p-$ Meshless cloud method," Computer Methods in Applied Mechanics and Engineering, vol. 139, no. 1-4, pp. 263-288, 1996.

[27] V. P. Nguyen, T. Rabczuk, S. Bordas, and M. Duflot, "Meshless methods: a review and computer implementation aspects," Mathematics and Computers in Simulation, vol. 79, no. 3, pp. 763-813, 2008.

[28] T. Belytschko, Y. Y. Lu, and L. Gu, "Element-free Galerkin methods," International Journal for Numerical Methods in Engineering, vol. 37, no. 2, pp. 229-256, 1994.

[29] Y. Y. Lu, T. Belytschko, and L. Gu, "A new implementation of the element free Galerkin method," Computer Methods in Applied Mechanics and Engineering, vol. 113, no. 3-4, pp. 397-414, 1994.

[30] S. N. Atluri and T. Zhu, "A new meshless local Petrov-Galerkin (MLPG) approach in computational mechanics," Computational Mechanics, vol. 22, no. 2, pp. 117-127, 1998.

[31] H. Lin and S. N. Atluri, "The meshless local Petrov-Galerkin (MLPG) method for solving incompressible Navier-Stokes equations," Computer Modeling in Engineering \& Sciences, vol. 2, no. 2, pp. 117-142, 2001.

[32] Q. W. Ma, "Meshless local Petrov-Galerkin method for twodimensional nonlinear water wave problems," Journal of Computational Physics, vol. 205, no. 2, pp. 611-625, 2005.

[33] Q. W. Ma, "MLPG method based on Rankine source solution for simulating nonlinear water waves," Computer Modeling in Engineering and Sciences, vol. 9, no. 2, pp. 193-209, 2005.

[34] Q. W. Ma and J. T. Zhou, "MLPG-R method for numerical simulation of 2D breaking waves," CMES-Computer Modeling in Engineering and Sciences, vol. 43, no. 3, pp. 277-303, 2009. 
[35] J. T. Zhou and Q. W. Ma, "MLPG method based on rankine source solution for modelling $3 \mathrm{D}$ breaking waves," CMES: Computer Modeling in Engineering \& Sciences, vol. 56, no. 2, pp. 179-210, 2010.

[36] N. Fathianpour, H. Hashemolhosseini, and M. Tabatabaie, "Application of geostatistical weights in solving problems governed by 2-D Poisson's equation using finite point method," in Computational Methods, pp. 1823-1828, Springer, Dordrecht, The Netherlands, 2006.

[37] S. H. Lee and Y. C. Yoon, "Meshfree point collocation method for elasticity and crack problems," International Journal for Numerical Methods in Engineering, vol. 61, no. 1, pp. 22-48, 2004.

[38] B. Boroomand, M. Najjar, and E. Oñate, "The generalized finite point method," Computational Mechanics, vol. 44, no. 2, pp. $173-$ 190, 2009.

[39] R. Löhner, C. Sacco, E. Oñate, and S. Idelsohn, "A finite point method for compressible flow," International Journal for Numerical Methods in Engineering, vol. 53, no. 8, pp. 1765-1779, 2002.

[40] E. Ortega, E. Oñate, and S. Idelsohn, "An improved finite point method for tridimensional potential flows," Computational Mechanics, vol. 40, no. 6, pp. 949-963, 2007.

[41] F. Perazzo, R. Löhner, and L. Perez-Pozo, "Adaptive methodology for meshless finite point method," Advances in Engineering Software, vol. 39, no. 3, pp. 156-166, 2008.

[42] N. R. Aluru, "A point collocation method based on reproducing kernel approximations," International Journal for Numerical Methods in Engineering, vol. 47, no. 6, pp. 1083-1121, 2000.

[43] N. R. Aluru and G. Li, "Finite cloud method: a true meshless technique based on fixed reproducing kernel approximation," International Journal for Numerical Methods in Engineering, vol. 50, no. 10, pp. 2373-2410, 2001.

[44] X. Jin, G. Li, and N. R. Aluru, "New approximations and collocation schemes in the finite cloud method," Computers \& Structures, vol. 83, no. 17-18, pp. 1366-1385, 2005.

[45] C. Lee, D. W. Kim, S. Park, H. Kim, C. Im, and H. Jung, "Point collocation mesh-free method using FMLSRKM for solving axisymmetric Laplace equation," IEEE Transactions on Magnetics, vol. 44, no. 6, pp. 1234-1237, 2008.

[46] S. W. Yang, Y. M. Wang, C. P. Wu, and H. T. Hu, "A meshless collocation method based on the differential reproducing kernel approximation," Computer Modeling in Engineering and Sciences, vol. 60, no. 1, pp. 1-39, 2010.

[47] N. J. Wu and T. K. Tsay, "A robust local polynomial collocation method," International Journal for Numerical Methods in Engineering, vol. 93, no. 4, pp. 355-375, 2013.

[48] N. J. Wu, T. K. Tsay, T. C. Yang, and H. Y. Chang, "Orthogonal grid generation of an irregular region using a local polynomial collocation method," Journal of Computational Physics, vol. 243, pp. 58-73, 2013.

[49] B. S. Chen, T. K. Tsay, C. W. Yang, and K. C. Chiang, "Regional connectivity in modified finite point method," Engineering Analysis with Boundary Elements, vol. 47, pp. 21-31, 2014.

[50] W. S. Tsung, S. C. Hsiao, T. Y. Huang, and N. J. Wu, "Simulation of propagation and run-up of landslide-induced waves using meshless method," Journal of Coastal Research, vol. 65, pp. 404409, 2013.

[51] T. Huang, S. Hsiao, and N. Wu, "Nonlinear wave propagation and run-up generated by subaerial landslides modeled using meshless method," Computational Mechanics, vol. 53, no. 2, pp. 203-214, 2014.
[52] N. J. Wu, T. K. Tsay, and Y. Y. Chen, "Generation of stable solitary waves by a piston-type wave maker," Wave Motion, vol. 51, no. 2, pp. 240-255, 2014.

[53] N. J. Wu, T. K. Tsay, Y. Y. Chen, and I. C. Tsu, "Simulation of 2D free-surface potential flows using a robust local polynomial collocation method," in Proceedings of APCOM \& ISCM, paper ID 1524, Singapore, 2013.

[54] H. Power and V. Barraco, "A comparison analysis between unsymmetric and symmetric radial basis function collocation methods for the numerical solution of partial differential equations," Computers \& Mathematics with Applications, vol. 43, no. 3-5, pp. 551-583, 2002.

[55] A. Sommerfeld, Mechanics of Deformable Bodies, Lectures on Theoretical Physics, Academic Press, New York, NY, USA, 1964.

[56] R. C. MacCormick and K. A. Fuch, "Wave forces on a pile: a diffraction theory," Tech. Memo. No 69, United States Army Corps of Engineers, 1954.

[57] J. F. Thompson, Z. U. A. Warsi, and C. W. Mastin, "Boundaryfitted coordinate systems for numerical solution of partial differential equations-a review," Journal of Computational Physics, vol. 47, no. 1, pp. 1-108, 1982.

[58] J. F. Thompson, Z. U. A. Warsi, and C. W. Mastin, Numerical Grid Generation: Foundation and Applications, North-Holland Publishing, Amsterdam, The Netherlands, 1985.

[59] T. K. Tsay and F. S. Hsu, "Numerical grid generation of an irregular region," International Journal for Numerical Methods in Engineering, vol. 40, no. 2, pp. 343-356, 1997.

[60] J. Wang and T.-K. Tsay, "Analytical evaluation and application of the singularities in boundary element method," Engineering Analysis with Boundary Elements, vol. 29, no. 3, pp. 241-256, 2005.

[61] J. Wang, T. K. Tsay, and F. R. Lin, "A short review of conformal grid generation in an irregular area," in Nonlinear Wave Dynamics: Selected Papers of the Symposium Held in Honor of Philip L-F Liu's 60th Birthday, pp. 199-222, 2008.

[62] T. K. Tsay, J. Wang, and Y. T. Huang, "Numerical generation and grid controls of boundary-fitted conformal grids in multiply connected regions," International Journal for Numerical Methods in Engineering, vol. 67, no. 8, pp. 1045-1062, 2006.

[63] T. Tsay and P. L.-F. Liu, "A finite element model for wave refraction and diffraction," Applied Ocean Research, vol. 5, no. 1, pp. 30-37, 1983.

[64] K. P. Yue, H. S. Chen, and C. C. Mei, "A hybrid element method for calculating three-dimensional water wave scattering," Tech. Rep. 215, Ralph M. Parsons Laboratory, Department of Civil Engineering, MIT, 1976.

[65] J. C. W. Berkhoff, Mathematical Models for Simple Harmonic Linear Water Waves, Wave Diffraction and Refraction, Pub. No. 163, Delft Hydraulic Laboratory, 1976.

[66] R. Smith and T. Sprinks, "Scattering of surface waves by a conical island," Journal of Fluid Mechanics, vol. 72, no. 2, pp. 373-384, 1975.

[67] P. Bettess and O. C. Zienkiewicz, "Diffraction and refraction of surface waves using finite and infinite elements," International Journal for Numerical Methods in Engineering, vol. 11, no. 8, pp. 1271-1290, 1977.

[68] J. R. Houston, "Modeling of short wave using the finite element method," in Proceedings of the 3rd International Conference on Finite Elements in Water Resources, vol. 5, pp. 181-195, 1980.

[69] J. R. Houston, "Combined refraction and diffraction of short waves using the finite element method," Applied Ocean Research, vol. 3, no. 4, pp. 163-170, 1981. 
[70] H. S. Chen and C. C. Mei, "Oscillations and wave forces in an offshore harbor,” Tech. Rep. 190, Ralph M. Parsons Laboratory for Water Resources and Hydrodynamics, M.I.T., Cambridge, Mass, USA, 1974.

[71] S. Homma, "On the behavior of seismic sea waves around circular island," Geophysical Magazine, vol. 21, pp. 199-208, 1950.

[72] J. J. Lee, "Wave-induced oscillations in harbors of arbitrary geometry," Journal of Fluid Mechanics, vol. 45, pp. 375-394, 1971.

[73] T. Tsay, "Linear surface waves over rotating fluids," Journal of Waterway, Port, Coastal and Ocean Engineering, vol. 117, no. 2, pp. 156-171, 1991.

[74] C. C. Mei and R. V. Petroni, "Wave in a harbor with protruding breakwaters," ASCE Journal of Waterway, Port, Coastal and Ocean Engineering, vol. 99, no. 2, pp. 209-229, 1973.

[75] J. Pedlosky, Geophysical Fluid Dynamics, Springer, New York, NY, USA, 3rd edition, 1987.

[76] H. S. Longuet-Higgins and E. D. Cokelet, "The deformation of steep surface waves on water. I. A numerical method of computation," Proceedings of the Royal Society. London. Series A. Mathematical, Physical and Engineering Sciences, vol. 350, no. 1660, pp. 1-26, 1976.

[77] S. T. Grilli, J. Skourup, and I. A. Svendsen, "An efficient boundary element method for nonlinear water waves," Engineering Analysis with Boundary Elements, vol. 6, no. 2, pp. 97-107, 1989.

[78] T. Ohyama and K. Nadaoka, "Development of a numerical wave tank for analysis of nonlinear and irregular wave field," Fluid Dynamics Research, vol. 8, no. 5-6, pp. 231-251, 1991.

[79] S. T. Grilli and P. Watts, "Modeling of waves generated by a moving submerged body. Applications to underwater landslides," Engineering Analysis with Boundary Elements, vol. 23, no. 8, pp. 645-656, 1999.

[80] S. T. Grilli, P. Guyenne, and F. Dias, "A fully nonlinear model for three-dimensional overturning waves over an arbitrary bottom," International Journal for Numerical Methods in Fluids, vol. 35, pp. 829-867, 2001.

[81] S. T. Grilli, S. Vogelmann, and P. Watts, "Development of a 3D numerical wave tank for modeling tsunami generation by underwater landslides," Engineering Analysis with Boundary Elements, vol. 26, no. 4, pp. 301-313, 2002.

[82] D. G. Goring, "Tsunamis-the propagation of long waves onto a shelf," Report KH-R-38, W. M. Keck Laboratory of Hydraulics and Water Resources, California Institute of Technology, Pasadena, Calif, USA, 1978.

[83] M. J. Boussinesq, “Théorie de l'intumescence liquide, appelée onde solitaire ou de translation, se propageant dans un canal rectangulaire," Comptes Rendus de l'Académie des Sciences, vol. 72, pp. 755-759, 1871.

[84] J. Fenton, "A ninth-order solution for the solitary wave," Journal of Fluid Mechanics, vol. 53, no. 2, pp. 257-271, 1972.

[85] D. Liu and P. Lin, "A numerical study of three-dimensional liquid sloshing in tanks," Journal of Computational Physics, vol. 227, no. 8, pp. 3921-3939, 2008. 


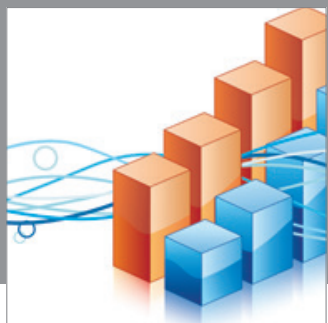

Advances in

Operations Research

mansans

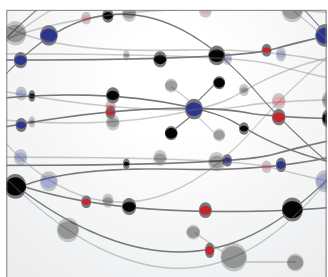

The Scientific World Journal
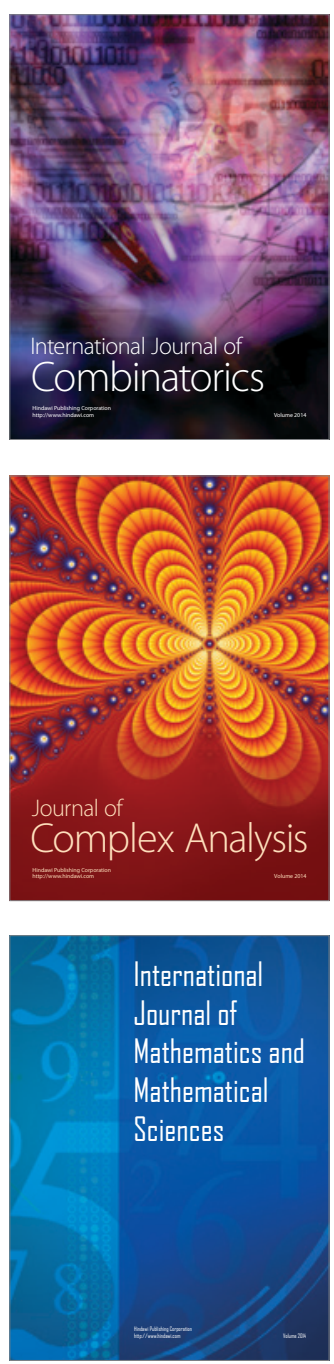
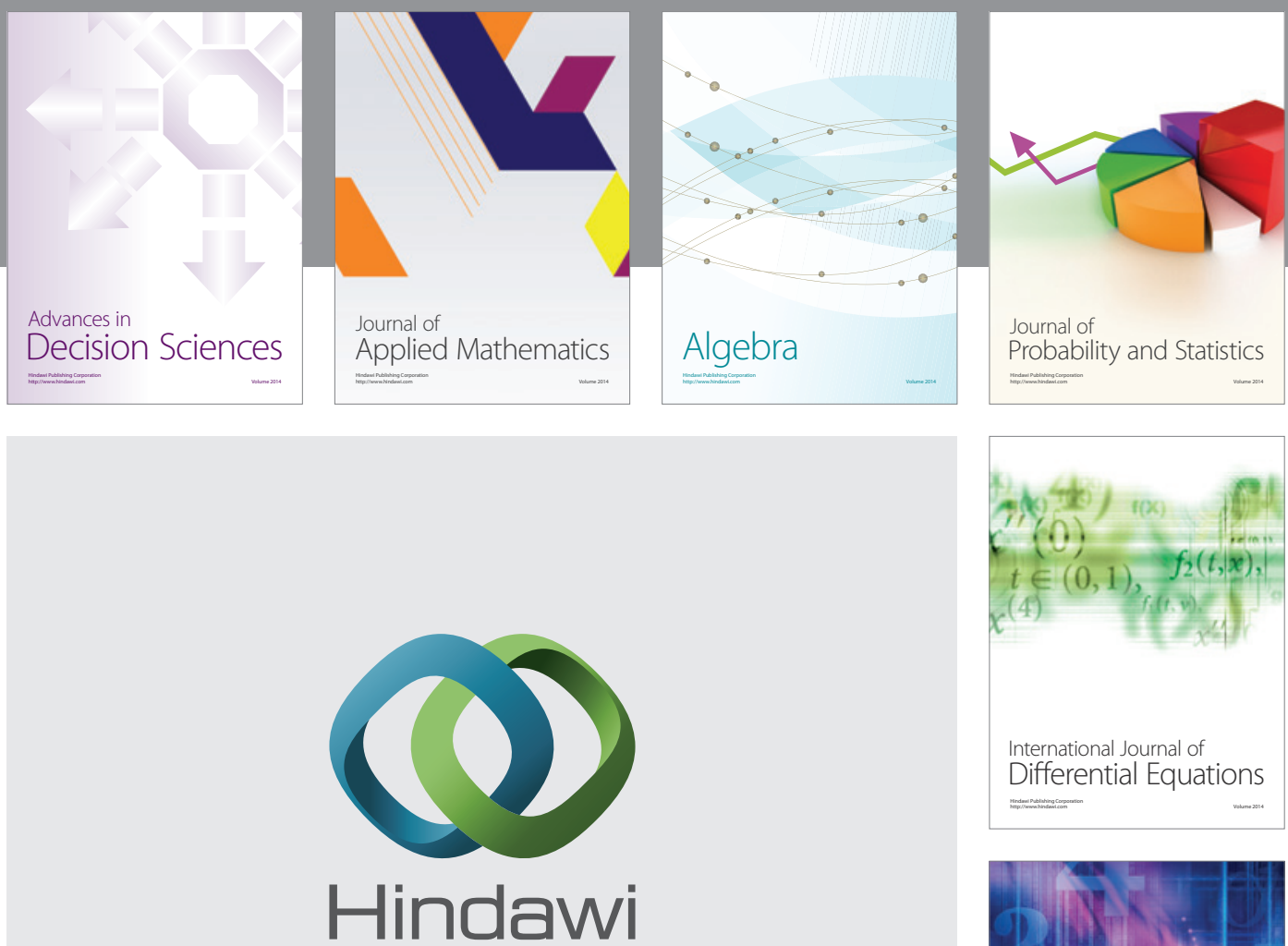

Submit your manuscripts at http://www.hindawi.com
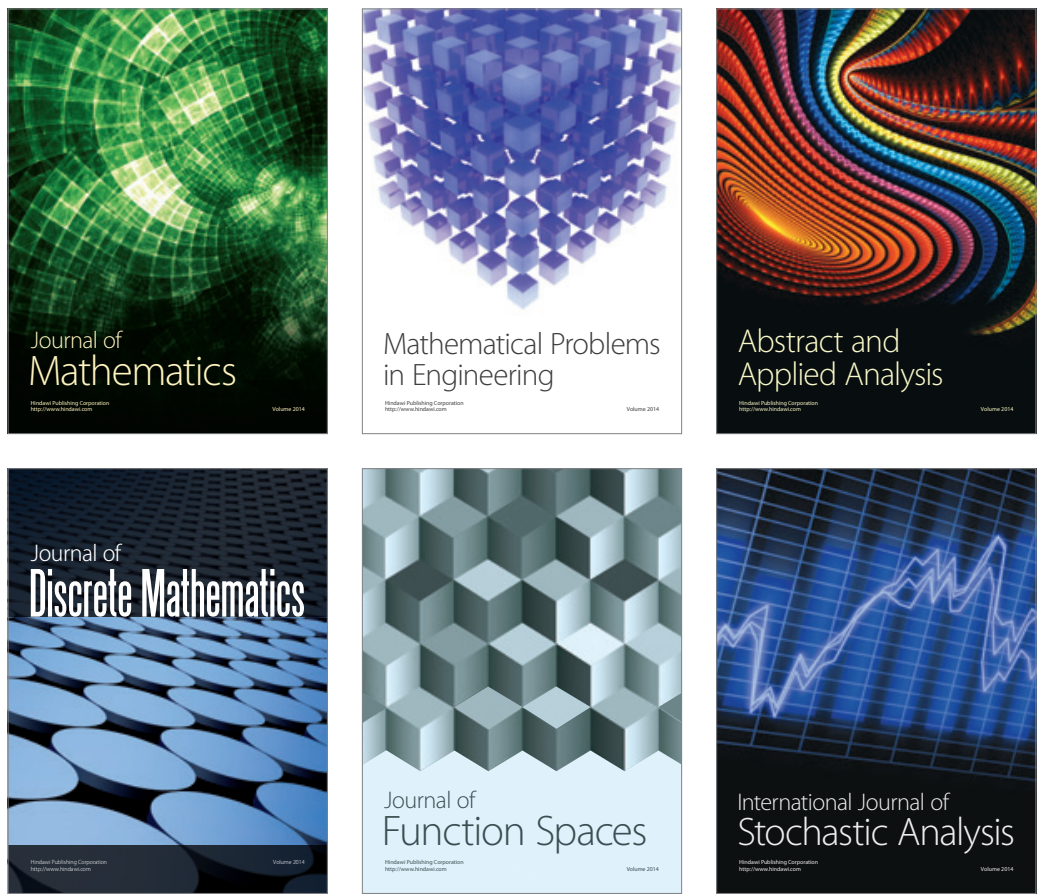

Journal of

Function Spaces

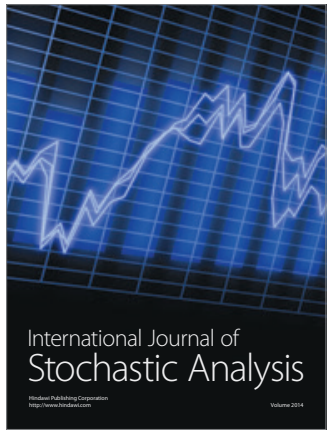

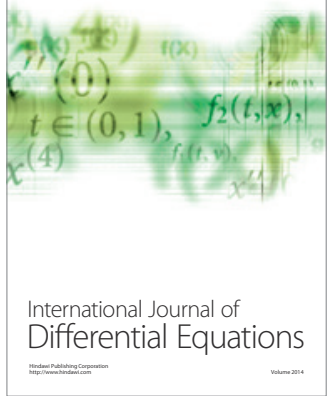
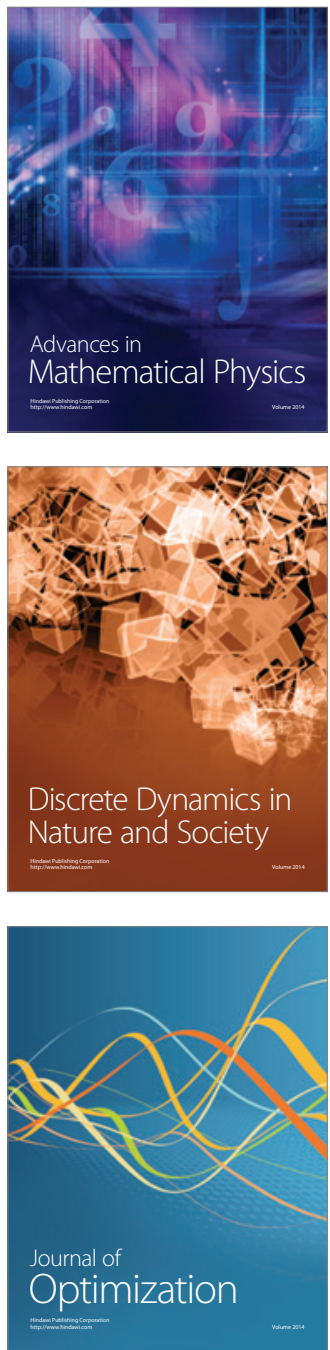